Research Article

\title{
Gradation of Continuity for Fuzzy Soft Mappings
}

\author{
Vildan Çetkin (iD) \\ Department of Mathematics, Kocaeli University, Umuttepe Campus, İmit 41001, Kocaeli, Turkey \\ Correspondence should be addressed to Vildan Çetkin; vildan.cetkin@kocaeli.edu.tr
}

Received 5 July 2021; Accepted 7 August 2021; Published 19 August 2021

Academic Editor: Ljubisa Kocinac

Copyright (c) 2021 Vildan Çetkin. This is an open access article distributed under the Creative Commons Attribution License, which permits unrestricted use, distribution, and reproduction in any medium, provided the original work is properly cited.

This paper is devoted to describe the notion of a parameterized degree of continuity for mappings between $L$-fuzzy soft topological spaces, where $L$ is a complete De Morgan algebra. The degrees of openness, closedness, and being a homeomorphism for the fuzzy soft mappings are also presented. The properties and characterizations of the proposed notions are pictured. Besides, the degree of continuity for a fuzzy soft mapping is unified with the degree of compactness and connectedness in a natural way.

\section{Introduction}

The theory of fuzzy sets which is a way of modeling real-life problems involves uncertainty, based on the degree of membership of an element to some sets. This idea has impressed many researchers working in diverse areas. Especially, the topology workers applied this idea to the gradation of openness and hence gave birth to the fuzzy topology [1]. The fuzzy-fuzzy case of the topology is the most compatible way of reflecting the gradation of belongingness [2]. So naturally, the notions of the degree of compactness, the degree of connectedness, the degree of separations, and so on have been considered. Later on, the similar argument has been considered for the mappings between fuzzy topological spaces and the degree of continuity, the degree of openness, and the degree of closedness for (fuzzy) mappings have been described [3-5]. The theory of soft sets is one of the other tools to model vague phenomena [6]. Also, the combination of fuzzy sets and soft sets gave birth to the fuzzy soft sets [7-9] and the basic idea of this new kind of sets depends on the parameterized degree of membership of an element to some sets. Nowadays, the studies depending on the soft sets and the fuzzy soft sets are increasing rapidly [10-13].

The idea of fuzzy softness (in fact, "parameterized gradation") is one of the appropriate tools for modeling of environmental and mathematical problems. On the other hand, the mappings play the key role in transforming the characteristics between structured spaces. Especially, the continuous mappings are worth to investigate since they preserve the several properties of the spaces endowed with topology. Motivated from this thinking, we found it reasonable to present a new theory which gives a more accurate and efficient way of transforming the characteristics between the fuzzy soft topological spaces depending on the parameters. Thus, as a continuation of the research studies [14-16], we describe the parameterized gradations of continuity, openness, and closedness for mappings between fuzzy soft topological spaces.

The content of this study is organized in the following manner: in Section 2, we present the notations and recall the elementary notions which are used throughout the study. In Section 3, we define the parameterized degree of the concepts of continuity, openness, closedness, and being homeomorphism for mappings transformed between fuzzy soft topological spaces. We investigate the parameterized graded extensions of the main properties and results known in general topology, for the proposed concepts. Additionally, we observe several characterizations of the described gradations with the help of the neighborhood systems and closure (interior) operators. At the end, we unify the parameterized graded continuity with the parameterized compactness (connectedness, respectively) degree.

\section{Preliminaries}

Throughout this paper, $X$ refers to a nonempty initial universe, $E$ and $K$ denotes the arbitrary nonempty sets 
viewed on the sets of parameters, and $L=\left(L, \vee, \wedge,{ }^{\prime}\right)$ denotes a complete De Morgan algebra with the smallest element $0_{L}$ and the largest element $1_{L}$. With the underlying lattice $L$, a mapping $A: X \longrightarrow L$ is said to be an $L$-fuzzy set on $X$ and by $L^{X}$, we denote the family of all $L$-fuzzy sets on $X$.

An element $\alpha$ in $L$ is said to be coprime if $\alpha \leq \beta \vee \gamma$ implies that $\alpha \leq \beta$ or $\alpha \leq \gamma \cdot M(L)$ denotes the collection of all coprime elements of $L$. We say $\alpha$ is way below (wedge below) $\beta$, in symbols, $\alpha \ll \beta,(\alpha \triangleleft \beta)$, if for every directed (arbitrary) subset $D \subseteq L, \vee D \geq \beta$ implies $\alpha \leq \gamma$ for some $\gamma \in D$. Clearly, if $\alpha \in L$ is coprime, then $\alpha \ll \beta$ if and only if $\alpha \triangleleft \beta$. Details for lattices can be found in [17].

The binary operation $\mapsto$ in the complete De Morgan algebra $L$ is given by $\alpha \mapsto \beta=\vee\{\gamma \in L \mid \alpha \wedge \gamma \leq \beta\}$.

For all $\alpha, \beta, \gamma, \delta \in L$ and $\left\{\alpha_{i}\right\},\left\{\beta_{i}\right\} \subseteq L$, the following properties are satisfied:

(1) $(\alpha \mapsto \beta) \geq \gamma$ iff $\alpha \wedge \gamma \leq \beta$

(2) $\alpha \mapsto \beta=1_{L}$ iff $\alpha \leq \beta$

(3) $\alpha \mapsto\left(\wedge_{i} \beta_{i}\right)=\wedge_{i}\left(\alpha \mapsto \beta_{i}\right)$ and $\left(\vee_{i} \alpha_{i}\right) \mapsto \beta=\wedge_{i}\left(\alpha_{i} \mapsto \beta\right)$

(4) $(\alpha \mapsto \gamma) \wedge(\gamma \mapsto \beta) \leq \alpha \mapsto \beta$

(5) $\alpha \leq \beta \Rightarrow \gamma \mapsto \alpha \leq \gamma \mapsto \beta$ and $\beta \mapsto \gamma \leq \alpha \mapsto \gamma$

(6) $(\alpha \mapsto \beta) \wedge(\gamma \mapsto \delta) \leq(\alpha \wedge \gamma) \mapsto(\beta \wedge \delta)$

Definition 1 (see [18]). A mapping $f: E \longrightarrow\left(L^{X}\right)^{E}$ is called an $L$-fuzzy soft set on $X$. This means that $f_{e}:=f(e): X$ $\longrightarrow L$ is an $L$-fuzzy set on $X$, for each parameter $e \in E$. Hence, an $L$-fuzzy soft set can be considered as the parameterized extended version of an $L$-fuzzy set. Intuitively, by a fuzzy soft set, one can describe the parameterized degree of belongingness.

From now on, we use the symbol $\left(L^{X}\right)^{E}$ to denote the collection of all $L$-fuzzy soft sets on $X$.

Definition 2 (see $[18,19])$. Let $f$ and $g$ be two $L$-fuzzy soft sets on $X$; then, the set operations are defined as follows:

(1) $f$ is an $L$-fuzzy soft subset of $g$ and written by $f \nsubseteq g$ if $f_{e} \leq g_{e}$, for each $e \in E$. $f$ and $g$ are called equal if $f \nsubseteq g$ and $g \nsubseteq f$.
(2) The union of $f$ and $g$ is an $L$-fuzzy soft set $h=f \sqcup g$, where $h_{e}=f_{e} \vee g_{e}$, for each $e \in E$.

(3) The intersection of $f$ and $g$ is an $L$-fuzzy soft set $h=f \sqcap g$, where $h_{e}=f_{e} \wedge g_{e}$, for each $e \in E$.

(4) The complement of $f$ is denoted by $f^{\prime}$, where $f^{\prime}: E \longrightarrow L^{X}$ is a mapping given by $f_{e}^{\prime}=\left(f_{e}\right)^{\prime}$, for each $e \in E$. Clearly $\left(f^{\prime}\right)^{\prime}=f$.

Definition 3 (see [18])

(1) An $L$-fuzzy soft set $f$ on $X$ is called a null (or empty) $L$-fuzzy soft set and denoted by $\widetilde{0}$, if $f_{e}(x)=0_{L}$, for each $e \in E, x \in X$.

(2) An $L$-fuzzy soft set $f$ on $X$ is called an absolute (or universal) $L$-fuzzy soft set and denoted by $\widetilde{1}$, if $f_{e}(x)=1_{L}$, for each $e \in E, x \in X$. Clearly $(\widetilde{1})^{\prime}=\widetilde{0}$ and $\widetilde{0}^{\prime}=\widetilde{1}$.

Definition 4 (see [20]). Let $x \in X$ and $\alpha: E \longrightarrow M(L)$ be a function. Then, the $L$-fuzzy soft set defined as follows is called an $L$-fuzzy soft point and denoted by $x^{\alpha}$.

$$
x_{e}^{\alpha}(y)=\left\{\begin{array}{ll}
\alpha(e), & \text { if } y=x, \\
0_{L}, & \text { otherwise, }
\end{array} \quad \text { for all } e \in E \text { and } y \in X .\right.
$$

An $L$-fuzzy soft point $x^{\alpha}$ is said to belong to an $L$-fuzzy soft set $f$ and denoted by $x^{\alpha} \in f$ if $\alpha(e) \leq f_{e}(x)$, for each $e \in E$.

The set of all nonzero coprime elements of $\left(L^{X}\right)^{E}$ is denoted by $M\left(\left(L^{X}\right)^{E}\right)$. It is noted that $M\left(\left(L^{X}\right)^{E}\right)$ is exactly the set of all $L$-fuzzy soft points.

Definition 5 (see [21]). Let $\varphi: X_{1} \longrightarrow X_{2}$ and $\psi: E_{1} \longrightarrow E_{2}$ be two functions, where $E_{1}$ and $E_{2}$ are parameter sets for the crisp sets $X_{1}$ and $X_{2}$, respectively. Then, the pair $(\varphi, \psi):=\varphi_{\psi}$ is called an $L$-fuzzy soft mapping from $\left(X_{1}, E_{1}\right)$ to $\left(X_{2}, E_{2}\right)$ :

(1) The image of $f \in\left(L^{X_{1}}\right)^{E_{1}}$ under $\varphi_{\psi}$, denoted by $\varphi_{\psi}(f)$, is an $L$-fuzzy soft set on $X_{2}$ defined by

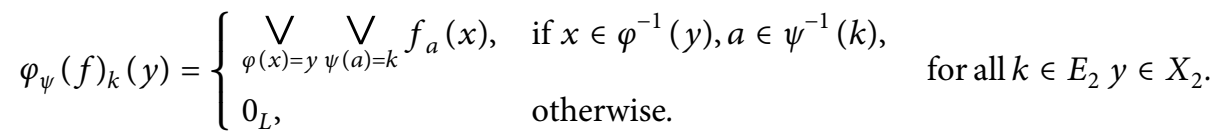

(2) The inverse image of $g \in\left(L^{X_{2}}\right)^{E_{2}}$ under $\varphi_{\psi}$, denoted by $\varphi_{\psi}^{-1}(g)$, is an $L$-fuzzy soft set on $X_{1}$ defined by $\varphi_{\psi}^{-1}(g)_{e}(x)=g_{\psi(e)}(\varphi(x)), \quad$ for all $e \in E_{1}, x \in X_{1}$.

(3) If $\varphi$ and $\psi$ are both injective (surjective or bijective, respectively), then $\varphi_{\psi}$ is said to be injective (surjective or bijective, respectively).
(4) If $\varphi_{\psi}:\left(X_{1}, E_{1}\right) \longrightarrow\left(X_{2}, E_{2}\right)$ and $\varphi_{\psi^{*}}^{*}:\left(X_{2}, E_{2}\right) \longrightarrow$ $\left(X_{3}, E_{3}\right)$ are soft mappings, then their composition $\left(\varphi_{\psi^{*}}^{*}{ }^{\circ} \varphi_{\psi}\right):\left(\mathrm{X}_{1}, \mathrm{E}_{1}\right) \longrightarrow\left(\mathrm{X}_{3}, \mathrm{E}_{3}\right)$ is defined by $\left(\varphi_{\psi^{*}}^{*}\right.$ $\left.{ }^{\circ} \varphi_{\psi}\right)=\left(\varphi^{* \circ} \varphi\right)_{\psi^{*} \circ \psi}$.

Definition 6 (see [14]). A mapping $\tau: K \longrightarrow L^{\left(L^{X}\right)^{E}}$ which satisfies the following certain axioms is called an $L$-fuzzy $(E, K)$-soft topology on $X$. 
(O1) $\tau_{k}(\widetilde{0})=\tau_{k}(\widetilde{1})=1_{L}$, for each $k \in K$.

(O2) $\tau_{k}(f \sqcap g) \geq \tau_{k}(f) \wedge \tau_{k}(g)$, for each $k \in K$ and for all $f, g \in\left(L^{X}\right)^{E}$.

(O3) $\tau_{k}\left(\sqcup_{i \in \Delta} f_{i}\right) \geq \wedge_{i \in \Delta} \tau_{k}\left(f_{i}\right)$, for all $\left\{f_{i}\right\}_{i \in \Delta} \subseteq\left(L^{X}\right)^{E}$ and for each $k \in K$.

Then, the pair $(X, \tau)$ is called an $L$-fuzzy $(E, K)$-soft topological space. The value $\tau_{k}(f)$ is interpreted as the degree of openness of an $L$-fuzzy soft set $f$ with respect to the parameter $k \in K$. So, the fuzzy soft topology can be thought as the gradation of parameterized degree of openness. Hence, the parameterized degree of closedness of a given $L$-fuzzy soft set is described by using the complement operator $\tau_{k}^{*}(f)=\tau_{k}\left(f^{\prime}\right)$.

Let $\tau^{1}$ and $\tau^{2}$ be $L$-fuzzy $(E, K)$-soft topologies on $X$. We say that $\tau^{1}$ is finer than $\tau^{2}\left(\tau^{2}\right.$ is coarser than $\left.\tau^{1}\right)$, denoted by $\tau^{2} \leq \tau^{1}$, if $\tau_{k}^{2}(f) \leq \tau_{k}^{1}(f)$ for each $k \in K$ and $f \in\left(L^{X}\right)^{E}$.

Definition 7 (see [14]). Let $\left(X_{1}, \tau^{1}\right)$ be an $L$-fuzzy $\left(E_{1}\right.$, $\left.K_{1}\right)$-soft topological space and $\left(X_{2}, \tau^{2}\right)$ be an $L$-fuzzy $\left(E_{2}\right.$, $K_{2}$ )-soft topological space. Let $\varphi: X_{1} \longrightarrow X_{2}, \psi: E_{1} \longrightarrow E_{2}$ and $\eta: K_{1} \longrightarrow K_{2}$ be crisp functions. Then, the fuzzy soft mapping $\varphi_{\psi, \eta}:\left(X_{1}, \tau^{1}\right) \longrightarrow\left(X_{2}, \tau^{2}\right)$ is called continuous if $\tau_{k}^{1}\left(\varphi_{\psi}^{-1}(h)\right) \geq \tau_{\eta(k)}^{2}(h)$ for all $h \in\left(L^{X_{2}}\right)^{E_{2}}$ and for all $k \in K_{1}$.

Definition 8 (see [20]). For a fixed fuzzy soft point $x^{\alpha}$, let the mappings $Q_{x^{\alpha}}: K \times\left(L^{X}\right)^{E} \longrightarrow L$ satisfy the following axioms for each $k \in K$ and $f, g \in\left(L^{X}\right)^{E}$ :

$$
\begin{aligned}
& \text { (FSQ1) } \mathbb{Q}_{x^{\alpha}}(k, \widetilde{1})=1_{L} \text { and } \mathscr{Q}_{x^{\alpha}}(k, \widetilde{0})=0_{L} \\
& \text { (FSQ2) If } Q_{x^{\alpha}}(k, f) \neq 0_{L} \text {, then } x^{\alpha} \nsubseteq f^{\prime} \\
& \text { (FSQ3) } \mathbb{Q}_{x^{\alpha}}(k, f \sqcap g)=Q_{x^{\alpha}}(k, f) \wedge \mathbb{Q}_{x^{\alpha}}(k, g) \\
& \text { (FSQ4) } \mathbb{Q}_{x^{\alpha}}(k, f)=\vee_{x^{\alpha} \nsubseteq g \sqsupseteq f^{\prime}} \wedge_{y^{\beta} \nsubseteq g^{\beta}} \mathscr{Q}_{y^{\beta}}\left(k, g^{\prime}\right)
\end{aligned}
$$

Then, the collection $\mathbb{Q}=\left\{\mathbb{Q}_{x^{\alpha}} \mid x^{\alpha} \in M\left(\left(L^{X}\right)^{E}\right)\right\}$ of maps presented above is called an $L$-fuzzy $(E, K)$-soft quasi-coincident neighborhood (shortly, $q$-nhood) system on $X$. The value $\mathbb{Q}_{x^{\alpha}}(k, f)$ represents the parameterized degree of being $q$-nhood of $f$ to the fuzzy soft point $x^{\alpha}$.

Proposition 1 (see [20]). Let $\tau$ be an L-fuzzy (E,K)-soft topology on X. Define the mapping $Q_{x^{\alpha}}^{\tau}: K \times\left(L^{X}\right)^{E} \longrightarrow L$ as follows: for each $k \in K, f \in\left(L^{X}\right)^{E}$ and $x^{\alpha} \in M\left(\left(L^{X}\right)^{E}\right)$,

$$
\mathcal{Q}_{x^{\alpha}}^{\tau}(k, f)=\underset{x^{\alpha} \nsubseteq g \sqsupseteq f^{\prime}}{\vee} \tau_{k}\left(g^{\prime}\right) .
$$

Then, the set of $\mathscr{Q}^{\tau}=\left\{\mathscr{Q}_{x^{\alpha}}^{\tau} \mid x^{\alpha} \in M\left(\left(L^{X}\right)^{E}\right)\right\}$ is an $L$-fuzzy $(E, K)$-soft $q$-nhood system on $X$, called induced $L$-fuzzy $(E, K)$-soft $q$-nhood system by $\tau$.

Definition 9. For a fixed fuzzy soft point $x^{\alpha}$, let the mappings $\mathcal{N}_{x^{\alpha}}: K \times\left(L^{X}\right)^{E} \longrightarrow L$ satisfy the following axioms for each $k \in K$ and $f, g \in\left(L^{X}\right)^{E}$.

$$
\begin{aligned}
& \text { (FSN1) } \mathcal{N}_{x^{\alpha}}(k, \widetilde{1})=1_{L} \text { and } \mathcal{N}_{x^{\alpha}}(k, \widetilde{0})=0_{L} \\
& \text { (FSN2) If } \mathcal{N}_{x^{\alpha}}(k, f) \neq 0_{L} \text {, then } x^{\alpha} \nsubseteq f \\
& \text { (FSN3) } \mathcal{N}_{x^{\alpha}}(k, f \sqcap g)=\mathcal{N}_{x^{\alpha}}(k, f) \wedge \mathcal{N}_{x^{\alpha}}(k, g) \\
& \text { (FSN4) } \mathcal{N}_{x^{\alpha}}(k, f)=\vee_{x^{\alpha} \nsubseteq g \nsubseteq f^{\prime}} \wedge_{y^{\beta} \triangleleft g} \mathcal{N}_{y^{\beta}}(k, g)
\end{aligned}
$$

Then, the collection of maps $\mathcal{N}=\left\{\mathcal{N}_{x^{\alpha}} \mid x^{\alpha} \in M\left(\left(L^{X}\right)^{E}\right)\right\}$ is called an $L$-fuzzy $(E, K)$-soft neighborhood (shortly, nhood) system on $X$.

Definition 10 (see [16]). A mapping cl: $K \times\left(L^{X}\right)^{E} \longrightarrow$ $L^{M\left(\left(L^{X}\right)^{E}\right)}$ is called an $L$-fuzzy $(E, K)$-soft closure operator on $X$ if it satisfies the following axioms for each $k \in K$ :

(C1) $c l(k, f)\left(x^{\alpha}\right)=\wedge_{\beta \triangleleft \alpha} c l(k, f)\left(x^{\beta}\right)$, for all $x^{\alpha} \in M$ $\left(\left(L^{X}\right)^{E}\right)$.

(C2) $c l(k, \widetilde{0})\left(x^{\alpha}\right)=0_{L}$ for any $x^{\alpha} \in M\left(\left(L^{X}\right)^{E}\right)$.

(C3) $c l(k, f)\left(x^{\alpha}\right)=1_{L}$ for any $x^{\alpha} \nsubseteq f$.

(C4) $c l(k, f \sqcup g)=c l(k, f) \vee c l(k, g)$.

(C5) $\quad \sigma_{a}\left(c l\left(k, \sqcup \sigma_{a}(c l(k, f))\right) \subseteq \sigma_{a}(c l(k, f))\right.$, where $\sigma_{a}(c l(k, f))=\left\{x^{\alpha} \in M\left(\left(L^{X}\right)^{E}\right) \mid c l(k, f)\left(x^{\alpha}\right) \geq a\right\}$.

The value $c l(k, f)\left(x^{\alpha}\right)$ is interpreted as the degree to which $x^{\alpha}$ belongs to the parameterized closure of the fuzzy soft set $f$.

Example 1 (see [16]). Let $c l: K \times\left(L^{X}\right)^{E} \longrightarrow\left(L^{X}\right)^{E}$ be the closure operator given in a parameterized $L$-soft topological space $(X, T)$. In this case, the mapping is $C l(k, f): M$ $\left(\left(L^{X}\right)^{E}\right) \longrightarrow 2$ defined in such a way that

$$
C l(k, f)\left(x^{\alpha}\right)= \begin{cases}1, & \text { if } x^{\alpha} \nsubseteq c l(k, f), \\ 0, & \text { otherwise, }\end{cases}
$$

satisfies the conditions of Definition 10.

Theorem 1 (see [16]). Let $\tau$ be an L-fuzzy (E, K)-soft topology on $X$. Then, the mapping C: $K \times\left(L^{X}\right)^{E} \longrightarrow L^{M\left(\left(L^{X}\right)^{E}\right)}$ defined by

$$
C(k, f)\left(x^{\alpha}\right)=\left(Q_{x^{\alpha}}^{\tau}\left(k, f^{\prime}\right)\right)^{\prime},
$$

is an L-fuzzy $(E, K)$-soft closure operator on $X$, which is called the L-fuzzy $(E, K)$-soft closure operator induced by $\tau$.

Definition 11. A mapping Int: $K \times\left(L^{X}\right)^{E} \longrightarrow L^{M\left(\left(L^{X}\right)^{E}\right)}$ is called an $L$-fuzzy $(E, K)$-soft interior operator on $X$ if it satisfies the following axioms for each $k \in K$ :

(I1) $\operatorname{Int}(k, f)\left(x^{\alpha}\right)=\wedge_{\beta \triangleleft \alpha} \operatorname{Int}(k, f)\left(x^{\beta}\right)$, for all $x^{\alpha} \in M$ $\left(\left(L^{X}\right)^{E}\right)$

(I2) $\operatorname{Int}(k, \widetilde{1})\left(x^{\alpha}\right)=1_{L}$ for any $x^{\alpha} \in M\left(\left(L^{X}\right)^{E}\right)$

(I3) $\operatorname{Int}(k, f)\left(x^{\alpha}\right)=0_{L}$ for any $x^{\alpha} \nsubseteq f$

(I4) $\operatorname{Int}(k, f \sqcap g)=\operatorname{Int}(k, f) \wedge \operatorname{Int}(k, g)$

(I5) $\delta_{a}(\operatorname{Int}(k, f)) \subseteq \delta_{a}\left(\operatorname{Int}\left(k, \sqcup \delta_{a}(\operatorname{Int}(k, f))\right)\right)$, where $\delta_{a}(\operatorname{Int}(k, f))=\left\{x^{\alpha} \in M\left(\left(L^{X}\right)^{E}\right) \mid\right.$ Int $(k, f)\left(x^{\alpha}\right) \leq$ a\}, for all $a \in L \backslash\left\{1_{L}\right\}$

The value $\operatorname{Int}(k, f)\left(x^{\alpha}\right)$ is interpreted as the degree to which $x^{\alpha}$ belongs to the parameterized interior of the fuzzy soft set $f$.

Theorem 2. Let $\tau$ be an L-fuzzy $(E, K)$-soft topology on $X$, and let $\mathcal{N}=\left\{\mathcal{N}_{x^{\alpha}} \mid x^{\alpha} \in M\left(\left(L^{X}\right)^{E}\right)\right\}$ be the nhood system induced by $\tau$. Define a mapping $I: K \times\left(L^{X}\right)^{E} \longrightarrow L^{M\left(\left(L^{X}\right)^{E}\right)}$ by 


$$
I(k, f)\left(x^{\alpha}\right)=N_{x^{\alpha}}(k, f) .
$$

Then, the mapping $I$ is an $L$-fuzzy $(E, K)$-soft interior operator on $X$, which is called the $L$-fuzzy $(E, K)$-soft interior operator induced by $\tau$.
Definition 12 (see [22]). Let $(X, \tau)$ be an $L$-fuzzy $(E, K)$-soft topological space. Then, identify a mapping $\operatorname{com}_{\tau}: K \longrightarrow$ $L^{\left(L^{X}\right)^{E}}$ in such a way that in order to picture the parameterized compactness degree,

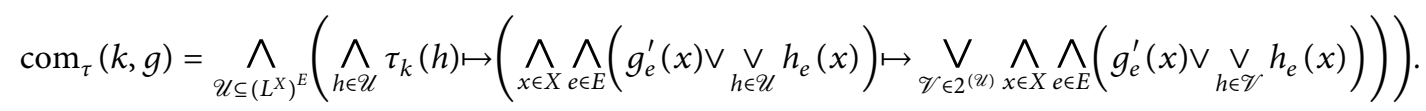

In this case, the value $\operatorname{com}_{\tau}(k, g)$ is interpreted as the compactness degree of $g \in\left(L^{X}\right)^{E}$ with respect to the parameter $k$. So, $g$ is said to be compact $L$-fuzzy soft set with respect to $k$ if $\operatorname{com}_{\tau}(k, g)=1_{L}$.
Definition 13 (see [16]). Let $(X, \tau)$ be an $L$-fuzzy $(E, K)$-soft topological space. Then, identify a mapping Con: $K \times$ $\left(L^{X}\right)^{E} \longrightarrow €$ by the following manner in order to describe the connectedness degree in such spaces:

$$
\operatorname{Con}(k, h)=\wedge\left\{\underset{x^{\alpha} \nsubseteq f}{\vee} C(k, g)\left(x^{\alpha}\right) \vee \underset{y^{\beta} \pm g}{\vee} C(k, f)\left(y^{\beta}\right) \mid f, g \in\left(L^{X}\right)^{E} \backslash\{\tilde{0}\}, h=f \sqcup g\right\} .
$$

In this case, the value $\operatorname{Con}(k, h)$ is said to be the connectedness degree of an $L$-fuzzy soft set $h$ with respect to $k$.

Theorem 3 (see [16]). Let $\tau$ be an L-fuzzy (E, K)-soft topology on $X$. Then, one can characterize the parameterized degree of connectedness of an $L$-fuzzy soft set $g \in\left(L^{X}\right)^{E}$ in the following way:

$$
\begin{aligned}
\operatorname{Con}(k, g)= & \wedge\left\{\left(\tau_{k}\left(u^{\prime}\right)\right)^{\prime} \vee\left(\tau_{k}\left(v^{\prime}\right)\right)^{\prime} \mid g \sqcap u \neq \widetilde{0},\right. \\
& \cdot g \sqcap v \neq \widetilde{0}, g \sqcap u \sqcap v=\widetilde{0} \text { and } g \nsubseteq u \sqcup v\} .
\end{aligned}
$$

\section{Degree of Continuity for Fuzzy Soft Mappings}

In this section, we identify the degrees of continuity, openness, closedness, and being a homeomorphism for a fuzzy soft mapping. Then, we study some of their characterizations by means of the q-nhood, nhood, interior, and closure operators. We also observe the elementary features of the proposed notions.

Definition 15. Let $\left(X_{1}, \tau^{1}\right)$ and $\left(X_{2}, \tau^{2}\right)$ be the $L$-fuzzy $\left(E_{1}, K_{1}\right)$-soft and $\left(E_{2}, K_{2}\right)$-soft topological spaces, respectively, and $\varphi_{\psi, \eta}:\left(X_{1}, \tau^{1}\right) \longrightarrow\left(X_{2}, \tau^{2}\right)$ be a soft mapping. Then, we define the following

(1) The parameterized degree of continuity for $\varphi_{\psi, \eta}$ is as follows: for all $k \in K_{1}$,

$$
\operatorname{Cont}\left(k, \varphi_{\psi, \eta}\right)=\wedge_{g \in\left(L^{X_{2}}\right)^{E_{2}}}\left(\tau_{\eta(k)}^{2}(g) \mapsto \tau_{k}^{1}\left(\varphi_{\psi}^{-1}(g)\right)\right) \text {. }
$$

The value Cont $\left(k, \varphi_{\psi, \eta}\right)$ represents to which $\varphi_{\psi, \eta}$ is continuous with respect to some parameters. Hence, the degree of continuity for $\varphi_{\psi, \eta}$ is computed by the formula $\operatorname{Cont}\left(\varphi_{\psi, \eta}\right)=\wedge_{k \in K_{1}} \operatorname{Cont}\left(k, \varphi_{\psi, \eta}\right)$.
(2) The parameterized degree of openness for $\varphi_{\psi, \eta}$ is as follows: for all $k \in K_{1}$,

$\operatorname{Open}\left(k, \varphi_{\psi, \eta}\right)=\wedge_{f \in\left(L^{X_{1}}\right)^{E_{1}}}\left(\tau_{k}^{1}(f) \mapsto \tau_{\eta(k)}^{2}\left(\varphi_{\psi}(f)\right)\right)$.

The value Open $\left(k, \varphi_{\psi, \eta}\right)$ represents to which $\varphi_{\psi, \eta}$ is open with respect to some parameters. Hence, the degree of openness for $\varphi_{\psi, \eta}$ is computed by the formula Open $\left(\varphi_{\psi, \eta}\right)=\wedge_{k \in K_{1}}$ Open $\left(k, \varphi_{\psi, \eta}\right)$.

(3) The parameterized degree of closedness for $\varphi_{\psi, \eta}$ is as follows: for all $k \in K_{1}$,

$\operatorname{Clos}\left(k, \varphi_{\psi, \eta}\right)=\bigwedge_{f \in\left(L^{X_{1}}\right)^{E_{1}}}\left(\tau_{k}^{1}\left(f^{\prime}\right) \mapsto \tau_{\eta(k)}^{2}\left(\varphi_{\psi}(f)^{\prime}\right)\right)$.

The value $\operatorname{Clos}\left(k, \varphi_{\psi, \eta}\right)$ represents to which $\varphi_{\psi, \eta}$ is a closed map with respect to some parameters. Hence, the degree of being a closed map for $\varphi_{\psi, \eta}$ is computed by the formula $\operatorname{Clos}\left(\varphi_{\psi, \eta}\right)=\wedge_{k \in K_{1}} \operatorname{Clos}\left(k, \varphi_{\psi, \eta}\right)$.

Definition 16. Let $\left(X_{1}, \tau^{1}\right)$ and $\left(X_{2}, \tau^{2}\right)$ be the $L$-fuzzy $\left(E_{1}, K_{1}\right)$-soft and $\left(E_{2}, K_{2}\right)$-soft topological spaces, respectively, and $\varphi_{\psi, \eta}:\left(X_{1}, \tau^{1}\right) \longrightarrow\left(X_{2}, \tau^{2}\right)$ be a bijective soft mapping. Then, the parameterized degree of being a homeomorphism for the soft mapping $\varphi_{\psi, \eta}$ is identified by $\operatorname{Hom}\left(k, \varphi_{\psi, \eta}\right)=\operatorname{Cont}\left(k, \varphi_{\psi, \eta}\right) \wedge \operatorname{Open}\left(k, \varphi_{\psi, \eta}\right)$. Hence, Hom $\left(\varphi_{\psi, \eta}\right)=\operatorname{Cont}\left(\varphi_{\psi, \eta}\right) \wedge \operatorname{Open}\left(\varphi_{\psi, \eta}\right)$.

\section{Remark 1}

(1) If the value Cont $\left(\varphi_{\psi, \eta}\right)=1_{L}$, then it is seen that $\tau_{k}^{1}\left(\varphi_{\psi, \eta}^{-1}(g)\right) \geq \tau_{\eta(k)}^{2}(g)$ for each $k \in K_{1}$ and for each $g \in\left(L^{X_{2}}\right)^{E_{2}}$ is satisfied. This gives us the definition of 
continuity for the mapping $\varphi_{\psi, \eta}:\left(X_{1}, \tau^{1}\right) \longrightarrow\left(X_{2}\right.$, $\left.\tau^{2}\right)$. Analogously, if $\operatorname{Open}\left(\varphi_{\psi, \eta}\right)=1_{L}$ or Clos $\left(\varphi_{\psi, \eta}\right)=1_{L}$, then (2) and (3) of Definition 15 are just the definitions of openness and closedness for the mapping $\varphi_{\psi, \eta}$, respectively.

(2) If $\varphi=i d_{X}, \psi=i d_{E}$, and $\eta=i d_{K}$ are the corresponding identity mappings, then $\varphi_{\psi, \eta}:(X, \tau) \longrightarrow$ $(X, \tau)$ is the identity soft mapping and $\operatorname{Cont}\left(\varphi_{\psi, \eta}\right)$ $=\operatorname{Open}\left(\varphi_{\psi, \eta}\right)=\operatorname{Hom}\left(\varphi_{\psi, \eta}\right)=1_{L}$.

Theorem 4. Let $\left(X_{i}, \tau^{i}\right)$ be the L-fuzzy $\left(E_{i}, K_{i}\right)$-soft topological spaces (where $i=1,2,3)$ and $\varphi_{\psi, \eta}:\left(X_{1}, \tau^{1}\right) \longrightarrow$
$\left(X_{2}, \tau^{2}\right)$ and $\delta_{\xi, \sigma}:\left(X_{2}, \tau^{2}\right) \longrightarrow\left(X_{3}, \tau^{3}\right)$ be the soft mappings. Then, the following is satisfied:

(1) $\operatorname{Cont}\left(k, \varphi_{\psi, \eta}\right)=\wedge_{g \in\left(L^{X_{2}}\right)^{E_{2}}}\left(\tau_{\eta(k)}^{2}\left(g^{\prime}\right) \mapsto \tau_{k}^{1}\left(\varphi_{\psi}^{-1}(g)^{\prime}\right)\right)$

(2) $\operatorname{Cont}\left(\delta_{\xi, \sigma}{ }^{\circ} \varphi_{\psi, \eta}\right) \geq \operatorname{Cont}\left(\varphi_{\psi, \eta}\right) \wedge \operatorname{Cont}\left(\delta_{\xi, \sigma}\right)$

(3) $\operatorname{Open}\left(\delta_{\xi, \sigma}{ }^{\circ} \varphi_{\psi, \eta}\right) \geq \operatorname{Open}\left(\varphi_{\psi, \eta}\right) \wedge \operatorname{Open}\left(\delta_{\xi, \sigma}\right)$

(4) $\operatorname{Clos}\left(\delta_{\xi, \sigma}{ }^{\circ} \varphi_{\psi, \eta}\right) \geq \operatorname{Clos}\left(\varphi_{\psi, \eta}\right) \wedge \operatorname{Clos}\left(\delta_{\xi, \sigma}\right)$

Proof. (2) If we consider Definition 15, then we obtain the following inequality:

$$
\begin{aligned}
& \operatorname{Cont}\left(k, \varphi_{\psi, \eta}\right) \wedge \wedge \operatorname{Cont}\left(\eta(k), \delta_{\xi, \sigma}\right) \\
& =\bigwedge_{g \in\left(L^{X_{2}}\right)^{E_{2}}}\left(\tau_{\eta(k)}^{2}(g) \mapsto \tau_{k}^{1}\left(\varphi_{\psi}^{-1}(g)\right)\right) \wedge \bigwedge_{h \in\left(L^{X_{3}}\right)^{E_{3}}}\left(\tau_{\sigma(\eta(k))}^{3}(h) \mapsto \tau_{\eta(k)}^{2}\left(\delta_{\xi}^{-1}(h)\right)\right) \\
& \leq \bigwedge_{h \in\left(L^{X_{3}}\right)^{E_{3}}}\left(\tau_{\eta(k)}^{2}\left(\delta_{\xi}^{-1}(h)\right) \mapsto \tau_{k}^{1}\left(\varphi_{\psi}^{-1}\left(\delta_{\xi}^{-1}(h)\right)\right)\right) \wedge \bigwedge_{h \in\left(L^{X_{3}}\right)^{E_{3}}}\left(\tau_{\sigma(\eta(k))}^{3}(h) \mapsto \tau_{\eta(k)}^{2}\left(\delta_{\xi}^{-1}(h)\right)\right) \\
& \leq \bigwedge_{h \in\left(L^{X_{3}}\right)^{E_{3}}}\left(\tau_{\eta(k)}^{2}\left(\delta_{\xi}^{-1}(h)\right) \mapsto \tau_{k}^{1}\left(\left(\delta_{\xi}^{\circ} \varphi_{\psi}\right)^{-1}(h)\right) \wedge\left(\tau_{\left(\sigma^{\circ} \eta\right)(k)}^{3}(h) \mapsto \tau_{\eta(k)}^{2}\left(\delta_{\xi}^{-1}(h)\right)\right)\right) \\
& \leq \bigwedge_{h \in\left(L^{X_{3}}\right)^{E_{3}}}\left(\tau_{\left(\sigma^{\circ} \eta\right)(k)}^{3}(h) \mapsto \tau_{k}^{1}\left(\left(\delta_{\xi}^{\circ} \varphi_{\psi}\right)^{-1}(h)\right)\right) \\
& \quad=\operatorname{Cont}\left(k, \delta_{\xi, \sigma}{ }^{\circ} \varphi_{\psi, \eta}\right),
\end{aligned}
$$

for any $k \in K_{1}$.

The above fact witnesses the proof.

The other conditions are similarly proved.

As it is well known in general topology, the composition of two homeomorphisms is again a homeomorphism. Then, by the above theorem, we get the following result for the gradation of homeomorphism.

Corollary 1. Let $\left(X_{i}, \tau^{i}\right)$ be the L-fuzzy $\left(E_{i}, K_{i}\right)$-soft topological spaces (where $i=1,2,3)$ and $\varphi_{\psi, \eta}:\left(X_{1}, \tau^{1}\right) \longrightarrow\left(X_{2}\right.$, $\left.\tau^{2}\right)$ and $\delta_{\xi, \sigma}:\left(X_{2}, \tau^{2}\right) \longrightarrow\left(X_{3}, \tau^{3}\right)$ be two bijective soft mappings. Then, $\operatorname{Hom}\left(\delta_{\xi, \sigma}{ }^{\circ} \varphi_{\psi, \eta}\right) \geq \operatorname{Hom}\left(\varphi_{\psi, \eta}\right) \wedge \operatorname{Hom}\left(\delta_{\xi, \sigma}\right)$ is satisfied.
Theorem 5. Let $\left(X_{i}, \tau^{i}\right)$ be the L-fuzzy $\left(E_{i}, K_{i}\right)$-soft topological spaces (where $i=1,2,3)$ and $\delta_{\xi, \sigma}:\left(X_{2}, \tau^{2}\right) \longrightarrow\left(X_{3}\right.$, $\left.\tau^{3}\right)$ be a soft mapping. If the fuzzy soft mapping $\varphi_{\psi, \eta}:\left(X_{1}\right.$, $\left.\tau^{1}\right) \longrightarrow\left(X_{2}, \tau^{2}\right)$ is surjective, then we get the following properties:

(1) $\operatorname{Open}\left(\delta_{\xi, \sigma}{ }^{\circ} \varphi_{\psi, \eta}\right) \wedge \operatorname{Cont}\left(\varphi_{\psi, \eta}\right) \leq \operatorname{Open}\left(\delta_{\xi, \sigma}\right)$

(2) $\operatorname{Clos}\left(\delta_{\xi, \sigma}{ }^{\circ} \varphi_{\psi, \eta}\right) \wedge \operatorname{Cont}\left(\varphi_{\psi, \eta}\right) \leq \operatorname{Clos}\left(\delta_{\xi, \sigma}\right)$

Proof. (1) $\left(\delta_{\xi}{ }^{\circ} \varphi_{\psi}\right)\left(\varphi_{\psi}^{-1}(\mathrm{~g})\right)=\delta_{\xi}(\mathrm{g})$, for each $g \in\left(L^{X_{2}}\right)^{E_{2}}$, is satisfied by the surjectivity of the soft mapping $\varphi_{\psi}$. Hence, we gain

$$
\begin{aligned}
& \operatorname{Open}\left(k, \delta_{\xi, \sigma}{ }^{\circ} \varphi_{\psi, \eta}\right) \wedge \operatorname{Cont}\left(k, \varphi_{\psi, \eta}\right) \\
& =\bigwedge_{f \in\left(L^{X_{1}}\right)^{E_{1}}}\left(\tau_{k}^{1}(f) \mapsto \tau_{\sigma(\eta(k))}^{3}\left(\delta_{\xi}{ }^{\circ} \varphi_{\psi}\right)(f)\right) \wedge \bigwedge_{g \in\left(L^{X_{2}}\right)^{E_{2}}}\left(\tau_{\eta(k)}^{2}(g) \mapsto \tau_{k}^{1}\left(\varphi_{\psi}^{-1}(g)\right)\right) \\
& \leq \bigwedge_{g \in\left(L^{X_{2}}\right)^{E_{2}}}\left(\tau_{k}^{1}\left(\varphi_{\psi}^{-1}(g)\right) \mapsto \tau_{\sigma(\eta(k))}^{3}\left(\delta_{\xi}^{\circ} \varphi_{\psi}\right)\left(\varphi_{\psi}^{-1}(g)\right)\right) \wedge \bigwedge_{g \in\left(L^{X_{2}}\right)^{E_{2}}}\left(\tau_{\eta(k)}^{2}(g) \mapsto \tau_{k}^{1}\left(\varphi_{\psi}^{-1}(g)\right)\right) \\
& =\bigwedge_{g \in\left(L^{X_{2}}\right)^{E_{2}}}\left\{\left(\tau_{k}^{1}\left(\varphi_{\psi}^{-1}(g)\right) \mapsto \tau_{\sigma(\eta(k))}^{3}\left(\delta_{\xi}^{\circ} \varphi_{\psi}\right)\left(\varphi_{\psi}^{-1}(g)\right)\right) \wedge\left(\tau_{\eta(k)}^{2}(g) \mapsto \tau_{k}^{1}\left(\varphi_{\psi}^{-1}(g)\right)\right)\right\} \\
& \leq \bigwedge_{g \in\left(L^{X_{2}}\right)^{E_{2}}}\left(\tau_{\eta(k)}^{2}(g) \mapsto \tau_{\sigma(\eta(k))}^{3}\left(\delta_{\xi}(g)\right)\right) \\
& =\operatorname{Open}\left(\eta(k), \delta_{\xi, \sigma}\right),
\end{aligned}
$$


for all $k \in K_{1}$.

Since $k \in K_{1}$ is arbitrary, we obtain the claimed inequality $\operatorname{Open}\left(\delta_{\xi, \sigma}{ }^{\circ} \varphi_{\psi, \eta}\right) \wedge \operatorname{Cont}\left(\varphi_{\psi, \eta}\right) \leq \operatorname{Open}\left(\delta_{\xi, \sigma}\right)$.

Theorem 6. Let $\left(X_{i}, \tau^{i}\right)$ be the L-fuzzy $\left(E_{i}, K_{i}\right)$-soft topological spaces (where $i=1,2,3)$ and $\varphi_{\psi, \eta}:\left(X_{1}, \tau^{1}\right) \longrightarrow\left(X_{2}\right.$, $\left.\tau^{2}\right)$ be a fuzzy soft mapping. If the fuzzy soft mapping $\delta_{\xi, \sigma}:\left(X_{2}, \tau^{2}\right) \longrightarrow\left(X_{3}, \tau^{3}\right)$ is injective, then we get the following properties:
(1) $\operatorname{Open}\left(\delta_{\xi, \sigma}{ }^{\circ} \varphi_{\psi, \eta}\right) \wedge \operatorname{Cont}\left(\delta_{\xi, \sigma}\right) \leq \operatorname{Open}\left(\varphi_{\psi, \eta}\right)$

(2) $\operatorname{Clos}\left(\delta_{\xi, \sigma}{ }^{\circ} \varphi_{\psi, \eta}\right) \wedge \operatorname{Cont}\left(\delta_{\xi, \sigma}\right) \leq \operatorname{Clos}\left(\varphi_{\psi, \eta}\right)$

Proof. (1) By considering the definitions, we get the following:

$$
\begin{aligned}
& \operatorname{Open}\left(\delta_{\xi, \sigma}{ }^{\circ} \varphi_{\psi, \eta}\right) \wedge \operatorname{Cont}\left(\delta_{\xi, \sigma}\right) \\
& =\bigwedge_{k \in K_{1}} \bigwedge_{f \in\left(L^{X_{1}}\right)^{E_{1}}}\left(\tau_{k}^{1}(f) \mapsto \tau_{\sigma(\eta(k))}^{3}\left(\left(\delta_{\xi}^{\circ} \varphi_{\psi}\right)(f)\right)\right) \wedge \bigwedge_{k^{*} \in K_{2}} \bigwedge_{h \in\left(L^{X_{3}}\right)^{E_{3}}}\left(\tau_{\sigma\left(k^{*}\right)}^{3}(h) \mapsto \tau_{k^{*}}^{2}\left(\delta_{\xi}^{-1}(h)\right)\right) \\
& \leq \bigwedge_{k \in K_{1}} \bigwedge_{f \in\left(L^{X_{1}}\right)^{E_{1}}}\left(\tau_{k}^{1}(f) \mapsto \tau_{\sigma(\eta(k))}^{3}\left(\left(\delta_{\xi}\left(\varphi_{\psi}(f)\right)\right) \wedge \bigwedge_{k \in K_{1}} \bigwedge_{g \in\left(L^{X_{2}}\right)^{E_{2}}}\left(\tau_{\sigma(\eta(k))}^{3}\left(\delta_{\xi}(g)\right) \mapsto \tau_{\eta(k)}^{2}\right)(g)\right)\right. \\
& \leq \bigwedge_{k \in K_{1}} \bigwedge_{f \in\left(L^{X_{1}}\right)^{E_{1}}}\left(\tau _ { k } ^ { 1 } ( f ) \mapsto \tau _ { \sigma ( \eta ( k ) ) } ^ { 3 } \left(\left(\delta_{\xi}\left(\varphi_{\psi}(f)\right)\right) \wedge \bigwedge_{k \in K_{1}} \bigwedge_{f \in\left(L^{X_{1}}\right)^{E_{1}}}\left(\tau_{\sigma(\eta(k))}^{3}\left(\delta_{\xi}\left(\varphi_{\psi}(f)\right)\right) \mapsto \tau_{\eta(k)}^{2}\left(\varphi_{\psi}(f)\right)\right)\right.\right. \\
& \leq \bigwedge_{k \in K_{1}} \bigwedge_{f \in\left(L^{X_{1}}\right)^{E_{1}}}\left(\left(\tau_{k}^{1}(f) \mapsto \tau_{\sigma(\eta(k))}^{3}\left(\delta_{\xi}\left(\varphi_{\psi}(f)\right)\right)\right) \wedge \tau_{\sigma(\eta(k))}^{3}\left(\delta_{\xi}\left(\varphi_{\psi}(f)\right) \mapsto \tau_{\eta(k)}^{2}\left(\varphi_{\psi}(f)\right)\right)\right) \\
& \leq \bigwedge_{k \in K_{1}} \bigwedge_{f \in\left(L^{X_{1}}\right)^{E_{1}}}\left(\tau_{k}^{1}(f) \mapsto \tau_{\eta(k)}^{2}\left(\varphi_{\psi}(f)\right)\right)=\operatorname{Open}\left(\varphi_{\psi, \eta}\right) .
\end{aligned}
$$

Theorem 7. Let $\left(X_{1}, \tau^{1}\right)$ and $\left(X_{2}, \tau^{2}\right)$ be the L-fuzzy $\left(E_{1}, K_{1}\right)$-soft and L-fuzzy $\left(E_{2}, K_{2}\right)$-soft topological spaces. If $\varphi_{\psi, \eta}:\left(X_{1}, \tau^{1}\right) \longrightarrow\left(X_{2}, \tau^{2}\right)$ is a bijective soft mapping, then we have

(1)

$\operatorname{Cont}\left(\varphi_{\psi, \eta}\right)=\wedge_{k \in K_{1}} \wedge_{f \in\left(L^{X_{1}}\right)^{E_{1}}}\left(\tau_{\eta(k)}^{2}\left(\varphi_{\psi}(f)\right) \mapsto \tau_{k}^{1}(f)\right)$

(2)

$\operatorname{Open}\left(\varphi_{\psi, \eta}\right)=\wedge_{k \in K_{1}} \wedge_{g \in\left(L^{X_{2}}\right)^{E_{2}}}\left(\tau_{k}^{1}\left(\varphi_{\psi}^{-1}(g)\right) \mapsto \tau_{\eta(k)}^{2}(g)\right)$
(3) $\operatorname{Cont}\left(\varphi_{\psi, \eta}^{-1}\right)=\operatorname{Open}\left(\varphi_{\psi, \eta}\right)=\operatorname{Clos}\left(\varphi_{\psi, \eta}\right)$

Proof. (1) Since the soft mapping $\varphi_{\psi}$ is bijective, then $\varphi_{\psi}^{-1}\left(\varphi_{\psi}(f)\right)=f$ for each $f \in\left(L^{X_{1}}\right)^{E_{1}}$ and $\varphi_{\psi}\left(\varphi_{\psi}^{-1}(g)\right)=g$ for each $g \in\left(L^{X_{2}}\right)^{E_{2}}$. By considering these facts, we gain the following:

$$
\begin{aligned}
\bigwedge_{k \in K_{1}} \bigwedge_{f \in\left(L^{X_{1}}\right)^{E_{1}}}\left(\tau_{\eta(k)}^{2}\left(\varphi_{\psi}(f)\right) \mapsto \tau_{k}^{1}(f)\right) & =\bigwedge_{k \in K_{1}} \bigwedge_{f \in\left(L^{X_{1}}\right)^{E_{1}}}\left(\tau_{\eta(k)}^{2}\left(\varphi_{\psi}(f)\right) \mapsto \tau_{k}^{1}\left(\varphi_{\psi}^{-1}\left(\varphi_{\psi}(f)\right)\right)\right) \\
& \geq \bigwedge_{k \in K_{1}} \bigwedge_{g \in\left(L^{X_{2}}\right)^{E_{2}}}\left(\tau_{\eta(k)}^{2}(g) \mapsto \tau_{k}^{1}\left(\varphi_{\psi}^{-1}(g)\right)\right) \\
& =\bigwedge_{k \in K_{1}} \bigwedge_{g \in\left(L^{X_{2}}\right)^{E_{2}}}\left(\tau_{\eta(k)}^{2}\left(\varphi_{\psi}\left(\varphi_{\psi}^{-1}(g)\right)\right) \mapsto \tau_{k}^{1}\left(\varphi_{\psi}^{-1}(g)\right)\right) \\
& \geq \bigwedge_{k \in K_{1}} \bigwedge_{f \in\left(L^{X_{1}}\right)^{E_{1}}}\left(\tau_{\eta(k)}^{2}\left(\varphi_{\psi}(f)\right) \mapsto \tau_{k}^{1}(f)\right) .
\end{aligned}
$$

This witnesses the fact that

$$
\operatorname{Cont}\left(\varphi_{\psi, \eta}\right)=\bigwedge_{k \in K_{1}} \bigwedge_{f \in\left(L^{X_{1}}\right)^{E_{1}}}\left(\tau_{\eta(k)}^{2}\left(\varphi_{\psi}(f)\right) \mapsto \tau_{k}^{1}(f)\right) .
$$

(2) It is similarly proved to that of (1).

(3) In order to obtain the proof, we will consider the equalities $\left(\varphi_{\psi}^{-1}\right)^{-1}(f)=\varphi_{\psi}(f)$ and $\varphi_{\psi}\left(f^{\prime}\right)=\left(\varphi_{\psi}(f)\right)^{\prime}$ (by the injectivity property) for each $f \in\left(L^{X_{1}}\right)^{E_{1}}$. So,

$$
\operatorname{Cont}\left(\varphi_{\psi}^{-1}\right)=\bigwedge_{k^{*} \in K_{2}} \bigwedge_{f \in\left(L^{X_{1}}\right)^{E_{1}}}\left(\tau_{\eta^{-1}\left(k^{*}\right)}^{1}(f) \mapsto \tau_{k^{*}}^{2}\left(\left(\varphi_{\psi}^{-1}\right)^{-1}\right)(f)\right)
$$

$$
\begin{aligned}
& =\bigwedge_{k^{*} \in K_{2}} \bigwedge_{f \in\left(L^{X_{1}}\right)^{E_{1}}}\left(\tau_{\eta^{-1}\left(k^{*}\right)}^{1}(f) \mapsto \tau_{k^{*}}^{2}\left(\varphi_{\psi}(f)\right)\right) \\
& =\bigwedge_{k \in K_{1}} \bigwedge_{f \in\left(L^{X_{1}}\right)^{E_{1}}}\left(\tau_{k}^{1}(f) \mapsto \tau_{\eta(k)}^{2}\left(\varphi_{\psi}(f)\right)=\operatorname{Open}\left(\varphi_{\psi, \eta}\right) .\right.
\end{aligned}
$$

In addition, we have 


$$
\begin{aligned}
\operatorname{Open}\left(\varphi_{\psi, \eta}\right) & =\wedge_{k \in K_{1}} \wedge_{f \in\left(L^{X_{1}}\right)^{E_{1}}}\left(\tau_{k}^{1}(f) \mapsto \tau_{\eta(k)}^{2}\left(\varphi_{\psi}(f)\right)\right. \\
& =\bigwedge_{k \in K_{1}} \wedge_{f \in\left(L^{X_{1}}\right)^{E_{1}}}\left(\tau_{k}^{1}\left(f^{\prime}\right) \mapsto \tau_{\eta(k)}^{2}\left(\varphi_{\psi}\left(f^{\prime}\right)\right)\right. \\
& =\wedge_{k \in K_{1}} \wedge_{f \in\left(L^{X_{1}}\right)^{E_{1}}}\left(\tau_{k}^{1}\left(f^{\prime}\right) \mapsto \tau_{\eta(k)}^{2}\left(\varphi_{\psi}(f)^{\prime}\right)\right. \\
& =\operatorname{Clos}\left(\varphi_{\psi, \eta}\right) .
\end{aligned}
$$

Corollary 2. Let $\left(X_{1}, \tau^{1}\right)$ and $\left(X_{2}, \tau^{2}\right)$ be an L-fuzzy $\left(E_{1}, K_{1}\right)$-soft and L-fuzzy $\left(E_{2}, K_{2}\right)$-soft topological spaces, respectively. If $\varphi_{\psi, \eta}:\left(X_{1}, \tau^{1}\right) \longrightarrow\left(X_{2}, \tau^{2}\right)$ is bijective, then the following characterizations are valid:

(1) $\operatorname{Hom}\left(\varphi_{\psi, \eta}\right)=\operatorname{Cont}\left(\varphi_{\psi, \eta}\right) \wedge \operatorname{Cont}\left(\varphi_{\psi, \eta}^{-1}\right)=\operatorname{Cont}\left(\varphi_{\psi, \eta}\right) \wedge$ $\operatorname{Clos}\left(\varphi_{\psi, \eta}\right)$

(2) $\operatorname{Hom}\left(\varphi_{\psi, \eta}\right)=\wedge_{k \in K_{1}} \wedge_{f \in\left(L^{X_{1}}\right)^{E_{1}}}\left(\tau_{\eta(k)}^{2}\left(\varphi_{\psi}(f)\right) \leftrightarrow \tau_{k}^{1}(f)\right)$
(3) $\operatorname{Hom}\left(\varphi_{\psi, \eta}\right)=\wedge_{k \in K_{1}} \wedge_{g \in\left(L^{X_{2}}\right)^{E_{2}}}\left(\tau_{k}^{1}\left(\varphi_{\psi}^{-1}(g)\right) \leftrightarrow \tau_{\eta(k)}^{2}(g)\right)$

Theorem 8. Let $\left(X_{1}, \tau^{1}\right)$ and $\left(X_{2}, \tau^{2}\right)$ be an L-fuzzy $\left(E_{1}\right.$, $\left.K_{1}\right)$-soft and L-fuzzy $\left(E_{2}, K_{2}\right)$-soft topological spaces, respectively. For any fuzzy soft mapping $\varphi_{\psi, \eta}:\left(X_{1}, \tau^{1}\right) \longrightarrow\left(X_{2}, \tau^{2}\right)$, the following characterizations are satisfied:

(1) $\operatorname{Cont}\left(\varphi_{\psi, \eta}\right)=\wedge_{k \in K_{1}} \wedge_{g \in\left(L^{X_{2}}\right)^{E_{2}}} \wedge_{x^{\alpha}}\left(\widehat{Q}_{\varphi_{\psi}\left(x^{\alpha}\right)}^{2}(\eta(k)), g\right.$ $\left.\mapsto Q_{x^{\alpha}}^{1}\left(k, \varphi_{\psi}^{-1}(g)\right)\right)$

(2) $\operatorname{Cont}\left(\varphi_{\psi, \eta}\right)=\wedge_{k \in K_{1}} \wedge_{g \in\left(L^{X_{2}}\right)^{E_{2}}} \quad \wedge_{x^{\alpha}}\left(\mathcal{N}_{\varphi_{\psi}\left(x^{\alpha}\right)}^{2}(\eta(k)), g\right.$ $\left.\mapsto \mathcal{N}_{x^{\alpha}}^{1}\left(k, \varphi_{\psi}^{-1}(g)\right)\right)$

(3) $\operatorname{Cont}\left(\varphi_{\psi, \eta}\right)=\wedge_{k \in K_{1}} \wedge_{g \in\left(L^{X_{2}}\right)^{E_{2}}} \wedge_{x^{\alpha}}\left(\operatorname{Int}^{2}(\eta(k)), g\left(\varphi_{\psi}\right.\right.$ $\left.\left.\left(x^{\alpha}\right)\right) \mapsto \operatorname{Int}^{1}\left(k, \varphi_{\psi}^{-1}(g)\right)\left(x^{\alpha}\right)\right)$

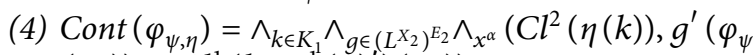
$\left.\left.\left(x^{\alpha}\right)\right) \mapsto C l^{1}\left(k, \varphi_{\psi}^{-1}(g)^{\prime}\right)\left(x^{\alpha}\right)\right)$

Proof. (1) By Proposition 1, $\varphi_{\psi}\left(x^{\alpha}\right) \nsubseteq h^{\prime} \sqsupseteq g^{\prime}$ implies $x^{\alpha} \nsubseteq \varphi_{\psi}^{-1}\left(h^{\prime}\right) \sqsupseteq \varphi_{\psi}^{-1}(g)^{\prime}$. Hence, we may obtain

$$
\begin{aligned}
& \bigwedge_{k \in K_{1}} \bigwedge_{g \in\left(L^{X_{2}}\right)^{E_{2}}} \bigwedge_{x^{\alpha}}\left({Q^{2}}_{\varphi_{\psi}\left(x^{\alpha}\right)}(\eta(k), g) \mapsto Q_{x^{\alpha}}^{1}\left(k, \varphi_{\psi}^{-1}(g)\right)\right) \\
& =\bigwedge_{k \in K_{1}} \bigwedge_{g \in\left(L^{X_{2}}\right)^{E_{2}}} \bigwedge_{x^{\alpha}}\left(\underset{\varphi_{\psi}\left(x^{\alpha}\right) \nsubseteq h^{\prime} \sqsupseteq g^{\prime}}{\vee} \tau_{\eta(k)}^{2}(h) \mapsto \underset{x^{\alpha} \nsubseteq w^{\prime} \sqsupseteq \varphi_{\psi}^{-1}(g)^{\prime}}{\vee} \tau_{k}^{1}(w)\right) \\
& =\bigwedge_{k \in K_{1}} \bigwedge_{g \in\left(L^{X_{2}}\right)^{E_{2}}} \bigwedge_{x^{\alpha}}\left(\underset{\varphi_{\psi}\left(x^{\alpha}\right) \nsubseteq h^{\prime} \sqsupseteq g^{\prime}}{\vee} \tau_{\eta(k)}^{2}(h) \mapsto \underset{x^{\alpha} \nsubseteq \varphi_{\psi}^{-1}}{\vee} \underset{\left(u^{\prime}\right) \sqsupseteq \varphi_{\psi}^{-1}(g)^{\prime}}{\vee} \tau_{k}^{1}\left(\varphi_{\psi}^{-1}(u)\right)\right) \\
& \geq \bigwedge_{k \in K_{1}} \bigwedge_{g \in\left(L^{X_{2}}\right)^{E_{2}}} \bigwedge_{x^{\alpha} \varphi_{\psi}\left(x^{\alpha}\right) \nsubseteq h^{\prime} \sqsupseteq g^{\prime}}\left(\tau_{\eta(k)}^{2}(h) \mapsto \tau_{k}^{1}\left(\varphi_{\psi}^{-1}(h)\right)\right) \\
& \geq \bigwedge_{k \in K_{1}} \bigwedge_{g \in\left(L^{X_{2}}\right)^{E_{2}}}\left(\tau_{\eta(k)}^{2}(g) \mapsto \tau_{k}^{1}\left(\varphi_{\psi}^{-1}(g)\right)\right)=\operatorname{Cont}\left(\varphi_{\psi, \eta}\right) .
\end{aligned}
$$

In order to prove the converse, consider the fact that $\tau_{k}(f)=\wedge_{x^{\alpha} \triangleleft f} Q_{x^{\alpha}}^{\tau}(k, f)$, for each $f \in\left(L^{X_{1}}\right)^{E_{1}}$ and $k \in K_{1}$.
Hence, $x^{\alpha} \nsubseteq \varphi_{\psi}^{-1}(g)^{\prime}$ implies $\varphi_{\psi}\left(x^{\alpha}\right) \nsubseteq g^{\prime}$. By these observations, we gain the following:

$$
\begin{aligned}
& \operatorname{Cont}\left(\varphi_{\psi, \eta}\right)=\bigwedge_{k \in K_{1}} \bigwedge_{g \in\left(L^{X_{2}}\right)^{E_{2}}}\left(\tau_{\eta(k)}^{2}(g) \mapsto \tau_{k}^{1}\left(\varphi_{\psi}^{-1}(g)\right)\right) \\
& =\bigwedge_{k \in K_{1}} \bigwedge_{g \in\left(L^{X_{2}}\right)^{E_{2}}}\left(\bigwedge_{y^{\beta} \triangleleft g} \mathbb{Q}_{y^{\beta}}^{2}(\eta(k), g) \mapsto \bigwedge_{x^{\alpha} \triangleleft \varphi_{\psi}^{-1}(g)} Q_{x^{\alpha}}^{1}\left(k, \varphi_{\psi}^{-1}(g)\right)\right) \\
& \geq \bigwedge_{k \in K_{1}} \bigwedge_{g \in\left(L^{X_{2}}\right)^{E_{2}}}\left(\bigwedge_{\varphi_{\psi}\left(x^{\alpha}\right) \triangleleft g} \mathbb{Q}_{\varphi_{\psi}\left(x^{\alpha}\right)}^{2}(\eta(k), g) \mapsto \bigwedge_{x^{\alpha} \triangleleft \varphi_{\psi}^{-1}(g)} Q_{x^{\alpha}}^{1}\left(k, \varphi_{\psi}^{-1}(g)\right)\right) \\
& \geq \bigwedge_{k \in K_{1}} \bigwedge_{g \in\left(L^{X_{2}}\right)^{E_{2}}} \bigwedge_{\varphi_{\psi}\left(x^{\alpha}\right) \triangleleft g}\left(Q_{\varphi_{\psi}\left(x^{\alpha}\right)}^{2}(\eta(k), g) \mapsto Q_{x^{\alpha}}^{1}\left(k, \varphi_{\psi}^{-1}(g)\right)\right)
\end{aligned}
$$

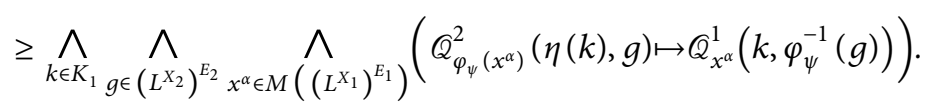


This completes the proof.

(2) It is similar to that of (1).

(3) and (4) proofs are obtained by using Theorems 1 and 2

Theorem 9. Let $\left(X_{1}, \tau^{1}\right)$ and $\left(X_{2}, \tau^{2}\right)$ be the L-fuzzy $\left(E_{1}, K_{1}\right)$-soft and L-fuzzy $\left(E_{2}, K_{2}\right)$-soft topological spaces. For the fuzzy soft mapping $\varphi_{\psi, \eta}:\left(X_{1}, \tau^{1}\right) \longrightarrow\left(X_{2}, \tau^{2}\right)$, the following properties are satisfied:

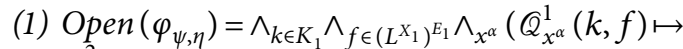
$\left.Q_{\varphi_{\psi}\left(x^{\alpha}\right)}^{2}\left(\eta(k), t \varphi_{\psi} n(f)\right)\right)$

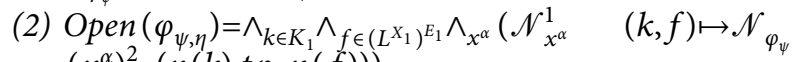
$\left.\left(x^{\alpha}\right)^{2}\left(\eta(k), t \varphi_{\psi} n(f)\right)\right)$

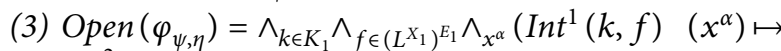
$\left.\operatorname{Int}^{2}\left(\eta(k), \varphi_{\psi}(f)\right)\left(\varphi_{\psi}\left(x^{\alpha}\right)\right)\right)$
(4) $\operatorname{Open}\left(\varphi_{\psi, \eta}\right)=\wedge_{k \in K_{1}} \wedge_{f \in\left(L^{X_{1}}\right)^{E_{1}}} \wedge_{x^{\alpha}}\left(C l^{1} \quad\left(k, f^{\prime}\right)\left(x^{\alpha}\right) \mapsto C l^{2}\right.$ $\left.\left(\eta(k), \varphi_{\psi}(f)^{\prime}\right)\left(\varphi_{\psi}\left(x^{\alpha}\right)\right)\right)$

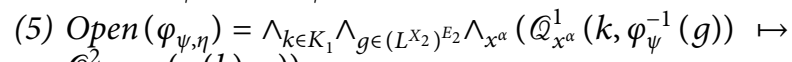
$\left.\widehat{Q}_{\varphi_{\psi}\left(x^{\alpha}\right)}^{2}(\eta(k), g)\right)$

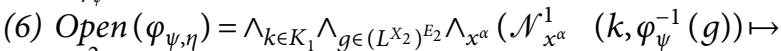 $\left.\mathcal{N}_{\varphi_{\psi}\left(x^{\alpha}\right)}^{2}(\eta(k), g)\right)$

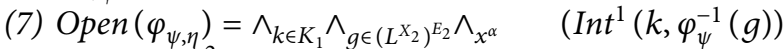
$\left.\left(x^{\alpha}\right) \mapsto \operatorname{Int}^{2}(\eta(k), g)\left(\varphi_{\psi}\left(x^{\alpha}\right)\right)\right)$

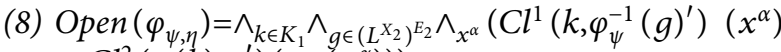
$\left.\mapsto C l^{2}\left(\eta(k), g^{\prime}\right)\left(\varphi_{\psi}\left(x^{\alpha}\right)\right)\right)$

Proof. (5) Let us first consider the following inequality:

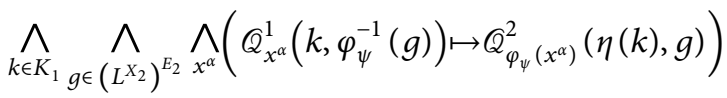

$$
\begin{aligned}
& =\bigwedge_{k \in K_{1}} \bigwedge_{g \in\left(L^{x_{2}}\right)^{E_{2}}} \bigwedge_{x^{\alpha}}\left(\underset{x^{\alpha} \ddagger h^{\prime} \sqsupseteq \varphi_{\psi}^{-1}(g)^{\prime}}{\bigvee} \tau_{k}^{1}(h) \mapsto \underset{\varphi_{\psi}\left(x^{\alpha}\right) \ddagger d^{\prime} \sqsupseteq g^{\prime}}{\vee} \tau_{\eta(k)}^{2}(d)\right) \\
& \geq \bigwedge_{k \in K_{1}} \bigwedge_{g \in\left(L^{X_{2}}\right)^{E_{2}}} \bigwedge_{x^{\alpha}}(\underset{x^{\alpha} \nsubseteq h^{\prime} \sqsupseteq \varphi_{\psi}^{-1}(g)^{\prime}}{\vee} \tau_{k}^{1}(h) \mapsto \underbrace{}_{\varphi_{\psi}\left(x^{\alpha}\right) \nsubseteq \varphi_{\psi}(u)^{\prime} \sqsupseteq g^{\prime}} \tau_{\eta(k)}^{2}\left(\varphi_{\psi}(u)\right)) \\
& \geq \bigwedge_{k \in K_{1}} \bigwedge_{g \in\left(L^{X_{2}}\right)^{E_{2}}} \wedge_{x^{\alpha}}\left(\underset{x^{\alpha} \nsubseteq h^{\prime} \sqsupseteq \varphi_{\psi}^{-1}(g)^{\prime}}{\bigvee} \tau_{k}^{1}(h) \mapsto{ }_{x^{\alpha} \nsubseteq u^{\prime} \sqsupseteq \varphi_{\psi}^{-1}(g)^{\prime}} \tau_{\eta(k)}^{2}\left(\varphi_{\psi}(u)\right)\right) \\
& \geq \bigwedge_{k \in K_{1}} \bigwedge_{g \in\left(L^{X_{2}}\right)^{E_{2}}} \bigwedge_{x^{\alpha} x^{\alpha} \nsubseteq h^{\prime} \sqsupseteq \varphi_{\psi}^{-1}(g)^{\prime}}\left(\tau_{k}^{1}(h) \mapsto \tau_{\eta(k)}^{2}\left(\varphi_{\psi}(h)\right)\right) \\
& \geq \bigwedge_{k \in K_{1}} \bigwedge_{f \in\left(L^{X_{1}}\right)^{E_{1}}}\left(\left(\tau_{k}^{1}(f) \mapsto \tau_{\eta(k)}^{2}\left(\varphi_{\psi}(f)\right)\right)=\operatorname{Open}\left(\varphi_{\psi, \eta}\right) .\right.
\end{aligned}
$$

In order to obtain the converse inequality, we will use the following fact:

$$
\wedge_{y^{\beta} \triangleleft \varphi_{\psi}(f)} Q_{y^{\beta}}^{2}\left(\eta(k), \varphi_{\psi}(f)\right)=\bigwedge_{\varphi_{\psi}\left(x^{\alpha}\right) \triangleleft \varphi_{\psi}(f)} \widehat{Q}_{\varphi_{\psi}\left(x^{\alpha}\right)}^{2}\left(\eta(k), \varphi_{\psi}(f)\right) .
$$

We may also consider

$$
\begin{aligned}
& \bigwedge_{k \in K_{1}} \bigwedge_{f \in\left(L^{X_{1}}\right)^{E_{1}}}\left(\bigwedge_{x^{\alpha} \triangleleft f} Q_{x^{\alpha}}^{1}\left(k, \varphi_{\psi}^{-1}\left(\varphi_{\psi}(f)\right)\right) \mapsto \bigwedge_{\varphi_{\psi}\left(x^{\alpha}\right) \triangleleft \varphi_{\psi}(f)} Q_{\varphi_{\psi}\left(x^{\alpha}\right)}^{2}\left(\eta(k), \varphi_{\psi}(f)\right)\right)
\end{aligned}
$$

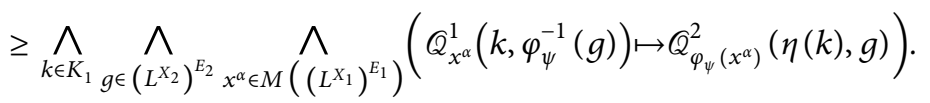

Now, let us choose an arbitrary $\gamma \in M(L)$ which satisfies

$$
\begin{aligned}
\gamma \triangleleft \bigwedge_{k \in K_{1}} \bigwedge_{g \in\left(L^{X_{2}}\right)^{E_{2}}} \bigwedge_{x^{\alpha} \in M\left(\left(L^{X_{1}}\right)^{E_{1}}\right)} & \cdot\left(\widehat{Q}_{x^{\alpha}}^{1}\left(k, \varphi_{\psi}^{-1}(g)\right) \mapsto \mathbb{Q}_{\varphi_{\psi}\left(x^{\alpha}\right)}^{2}(\eta(k), g)\right) .
\end{aligned}
$$

Therefore, $\quad \gamma \leq\left(\mathbb{Q}_{x^{\alpha}}^{1}\left(k, \varphi_{\psi}^{-1}(g)\right) \mapsto \mathbb{Q}_{\varphi_{\psi}\left(x^{\alpha}\right)}^{2}(\eta(k), g)\right)$ is satisfied for all $k \in K_{1}, g \in\left(L^{X_{2}}\right)^{E_{2}}$ and for all $x^{\alpha} \in M$ $\left(\left(L^{X_{1}}\right)^{E_{1}}\right)$. By the implication operator properties, we get $\gamma \wedge Q_{x^{\alpha}}^{1}\left(k, \varphi_{\psi}^{-1}(g)\right) \leq Q_{\varphi_{\psi}}^{2}\left(x^{\alpha}\right)(\eta(k), g)$. For all $f \in\left(L^{X_{1}}\right)^{E_{1}}$ with $\varphi_{\psi}\left(x^{\alpha}\right) \triangleleft \varphi_{\psi}(f)$, we obtain $\alpha(e) \leq \varphi_{\psi}(f)_{\psi(e)}(f(x))$. Then, there exist $z \in X$ and $e^{*} \in E$ such that $\varphi(x)=\varphi(z)$ 
and $\psi(e)=\psi\left(e^{*}\right)$ and also $\alpha\left(e^{*}\right) \leq f_{e^{*}}(z)$. This implies $z^{\alpha} \nsubseteq f^{\prime}$. From

$$
\begin{aligned}
\gamma \wedge & \wedge Q_{x^{\alpha} \nsubseteq f^{\prime}}^{1}\left(k, \varphi_{\psi}^{-1}\left(\varphi_{\psi}(f)\right)\right) \\
& \leq \gamma \wedge Q_{z^{\alpha}}^{1}\left(k, \varphi_{\psi}^{-1}\left(\varphi_{\psi}(f)\right)\right) \\
& \leq Q_{\varphi_{\psi}}^{2}\left(z^{\alpha}\right) \\
& \left(\eta(k), \varphi_{\psi}(f)\right)=Q_{\varphi_{\psi}\left(x^{\alpha}\right)}^{2}\left(\eta(k), \varphi_{\psi}(f)\right),
\end{aligned}
$$

we gain $\gamma \wedge \wedge_{x^{\alpha} \notin f^{\prime}} Q_{x^{\alpha}}^{1}\left(k, \varphi_{\psi}^{-1}\left(\varphi_{\psi} \quad(f)\right)\right) \leq \wedge_{\varphi_{\psi}\left(x^{\alpha}\right) \notin \varphi_{\psi}}(f)^{\prime}$ $Q_{\varphi_{\psi}\left(x^{\alpha}\right)}^{2}\left(\eta(k), \varphi_{\psi}(f)\right)$. So we get $\gamma \leq\left(\wedge_{x^{\alpha} \ddagger f^{\prime}} Q_{x^{\alpha}}^{1}\left(k, \varphi_{\psi}^{-1}\right.\right.$ $\left.\left.\left(\varphi_{\psi}(f)\right)\right) \mapsto t \wedge_{\varphi_{\psi}\left(x^{\alpha}\right) \nsubseteq \varphi_{\psi}(f)^{\prime}} n Q_{\varphi_{\psi}\left(x^{\alpha}\right)}^{2} q\left(\eta(k), \varphi_{\psi}(f)\right)\right)$. Hence, we also have

$$
\begin{gathered}
\gamma \leq \bigwedge_{k \in K_{1}} \bigwedge_{f \in\left(L^{X_{1}}\right)^{E_{1}}}\left(\bigwedge_{x^{\alpha} \nsubseteq f^{\prime}} Q_{x^{\alpha}}^{1}\left(k, \varphi_{\psi}^{-1}\left(\varphi_{\psi}(f)\right)\right) \mapsto\right. \\
\left.\cdot \bigwedge_{\varphi_{\psi}\left(x^{\alpha}\right) \nsubseteq \varphi_{\psi}(f)^{\prime}} Q_{\varphi_{\psi}\left(x^{\alpha}\right)}^{2}\left(\eta(k), \varphi_{\psi}(f)\right)\right) .
\end{gathered}
$$

Since the arbitrariness of $\gamma$, we gain

$$
\begin{aligned}
& \bigwedge_{k \in K_{1}} \bigwedge_{f \in\left(L^{X_{1}}\right)^{E_{1}}}\left(\bigwedge_{x^{\alpha} \nsubseteq f^{\prime}} \widehat{Q}_{x^{\alpha}}^{1}\left(k, \varphi_{\psi}^{-1}\left(\varphi_{\psi}(f)\right)\right) \mapsto \bigwedge_{\varphi_{\psi}\left(x^{\alpha}\right) \nsubseteq \varphi_{\psi}(f)^{\prime}} Q_{\varphi_{\psi}\left(x^{\alpha}\right)}^{2}\left(\eta(k), \varphi_{\psi}(f)\right)\right) \\
& \geq \bigwedge_{k \in K_{1}} \bigwedge_{g \in\left(L^{X_{2}}\right)^{E_{2}}} \bigwedge_{x^{\alpha} \in M}\left(\left(L^{X_{1}}\right)^{E_{1}}\right) \\
& \left(Q_{x^{\alpha}}^{1}\left(k, \varphi_{\psi}^{-1}(g)\right) \mapsto Q_{\varphi_{\psi}\left(x^{\alpha}\right)}^{2}(\eta(k), g)\right) .
\end{aligned}
$$

Since $f \nsubseteq \varphi_{\psi}^{-1}\left(\varphi_{\psi}(f)\right)$, for all $f \in\left(L^{X_{1}}\right)^{E_{1}}$, we obtain the following inequality:

$$
\begin{aligned}
& \operatorname{Open}\left(\varphi_{\psi, \eta}\right)=\bigwedge_{k \in K_{1}} \bigwedge_{f \in\left(L^{X_{1}}\right)^{E_{1}}}\left(\tau_{k}^{1}(f) \mapsto \tau_{\eta(k)}^{2}\left(\varphi_{\psi}(f)\right)\right)
\end{aligned}
$$

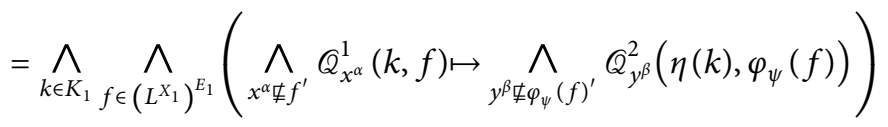

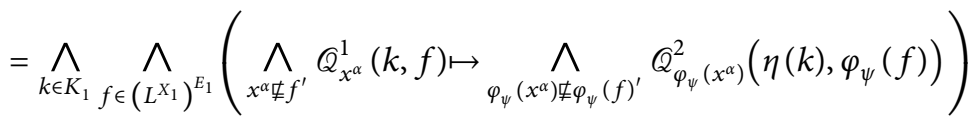

$$
\begin{aligned}
& \geq \bigwedge_{k \in K_{1}} \bigwedge_{f \in\left(L^{X_{1}}\right)^{E_{1}}}\left(\bigwedge_{x^{\alpha} \ddagger f^{\prime}}{Q_{x^{\alpha}}^{1}}^{1}\left(k, \varphi_{\psi}^{-1}\left(\varphi_{\psi}(f)\right)\right) \mapsto \bigwedge_{\varphi_{\psi}\left(x^{\alpha}\right) \nsubseteq \varphi_{\psi}(f)^{\prime}}{Q_{\varphi_{\psi}}^{2}\left(x^{\alpha}\right)}\left(\eta(k), \varphi_{\psi}(f)\right)\right)
\end{aligned}
$$

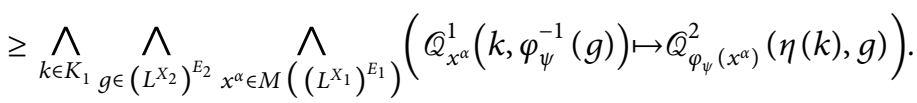

Hence we obtain the desired result.

$\operatorname{com}_{\tau^{1}}(k, f) \wedge \operatorname{Cont}\left(k, \varphi_{\psi, \eta}\right) \leq \operatorname{com}_{\tau^{2}}\left(\eta(k), \varphi_{\psi}(f)\right)$,

By using Theorems 1 and 2, and also by considering some similar discussion, one can prove the other claims of the theorem.

Theorem 10. Let $\left(X_{1}, \tau^{1}\right)$ and $\left(X_{2}, \tau^{2}\right)$ be the L-fuzzy $\left(E_{1}, K_{1}\right)$-soft and L-fuzzy $\left(E_{2}, K_{2}\right)$-soft topological spaces. For the fuzzy soft mapping $\varphi_{\psi, \eta}:\left(X_{1}, \tau^{1}\right) \longrightarrow\left(X_{2}, \tau^{2}\right)$, we have the following result: for all $k \in K_{1}$ and $f \in\left(L^{X_{1}}\right)^{E_{1}}$.

Proof. Let us choose an arbitrary $\beta \in M(L)$ such that $\beta \triangleleft\left(\operatorname{com}_{\tau^{1}}(k, f) \wedge \operatorname{Cont}\left(k, \varphi_{\psi, \eta}\right)\right)$. By the below wedge operation property, we have that

$$
\begin{aligned}
\beta \triangleleft \operatorname{Cont}\left(k, \varphi_{\psi, \eta}\right) & =\bigwedge_{g \in\left(L^{X_{2}}\right)^{E_{2}}}\left(\tau_{\eta(k)}^{2}(g) \mapsto \tau_{k}^{1}\left(\left(\varphi_{\psi}^{-1}(g)\right)\right)\right. \\
\beta \triangleleft \operatorname{com}_{\tau^{1}}(k, f) & =\bigwedge_{\mathscr{U} \subseteq\left(L^{X_{1}}\right)^{E_{1}}}\left\{\left(\bigwedge_{g \in \mathscr{U}} \tau_{k}^{1}(g) \wedge \bigwedge_{x \in X_{1}} \bigwedge_{e \in E_{1}}\left(f_{e}^{\prime}(x) \vee \bigvee_{g \in \mathcal{U}} h_{e}(x)\right)\right) \mapsto \bigvee_{\left.\mathscr{V} \in 2^{(\mathcal{U}}\right)} \bigwedge_{x \in X_{1}} \bigwedge_{e \in E_{1}}\left(f_{e}^{\prime}(x) \vee \bigvee_{g \in \mathscr{V}} g_{e}(x)\right)\right\}
\end{aligned}
$$


10

Journal of Mathematics

Hence for any $g \in\left(L^{X_{2}}\right)^{E_{2}}$ and for any $\mathcal{U} \subseteq\left(L^{X_{1}}\right)^{E_{1}}$, we gain

$$
\begin{aligned}
& \beta \leq\left(\tau_{\eta(k)}^{2}(g) \mapsto \tau_{k}^{1}\left(\varphi_{\psi}^{-1}(g)\right)\right), \\
& \beta \leq\left(\bigwedge_{g \in \mathscr{U}} \tau_{k}^{1}(g) \wedge \bigwedge_{x \in X_{1}} \bigwedge_{e \in E_{1}}\left(f_{e}^{\prime}(x) \bigvee \underset{g \in \mathscr{U}}{\vee} h_{e}(x)\right)\right) \mapsto \bigvee_{\mathscr{V} \in 2^{(\mathcal{C})}} \bigwedge_{x \in X_{1}} \bigwedge_{e \in E_{1}}\left(f_{e}^{\prime}(x) \bigvee \bigvee_{g \in \mathscr{V}} g_{e}(x)\right) .
\end{aligned}
$$

By considering the implication properties, we have

$$
\begin{array}{r}
\beta \wedge \tau_{\eta(k)}^{2}(g) \leq \tau_{k}^{1}\left(\varphi_{\psi}^{-1}(g)\right), \quad \text { for any } g \in\left(L^{X_{2}}\right)^{E_{2}}, \\
\beta \wedge\left(\bigwedge_{g \in \mathscr{U}} \tau_{k}^{1}(g) \wedge \bigwedge_{x \in X_{1}} \bigwedge_{e \in E_{1}}\left(f_{e}^{\prime}(x) \vee \bigvee_{g \in \mathscr{U}} h_{e}(x)\right)\right) \leq \bigvee_{\mathscr{V} \in 2^{(\mathcal{U})}} \bigwedge_{x \in X_{1}} \bigwedge_{e \in E_{1}}\left(f_{e}^{\prime}(x) \bigvee \bigvee_{g \in \mathscr{V}} g_{e}(x)\right)
\end{array}
$$

In order to complete the proof, it is necessary to show that

$$
\begin{aligned}
\beta & \leq \operatorname{com}_{\tau^{2}}\left(\eta(k), \varphi_{\psi}(f)\right) \\
& =\bigwedge_{\mathscr{W} \subseteq\left(L^{X_{2}}\right)^{E_{2}}}\left\{\left(\bigwedge_{w \in \mathscr{W}} \tau_{\eta(k)}^{2}(w) \wedge \bigwedge_{y \in X_{2}} \bigwedge_{e^{*} \in E_{2}}\left(\varphi_{\psi}(f)_{e^{*}}(y)^{\prime} \vee \bigvee_{w \in \mathscr{W}} w_{e^{*}}(y)\right)\right) \mapsto \bigvee_{\mathscr{D} \in 2^{(\mathscr{W})}} \bigwedge_{y \in X_{2}} \bigwedge_{e^{*} \in E_{2}}\left(\varphi_{\psi}(f)_{e^{*}}^{\prime}(y) \vee \bigvee_{w \in \mathscr{D}}^{\bigvee} w_{e^{*}}(y)\right)\right\} .
\end{aligned}
$$

Let $\varphi_{\psi}^{-1}(\mathscr{W})=\left\{\varphi_{\psi}^{-1}(w) \mid w \in \mathscr{W}\right\} \subseteq\left(L^{X_{1}}\right)^{E_{1}}$. Hence, we have the following facts:

$$
\begin{aligned}
& \beta \wedge\left(\bigwedge_{w \in \mathscr{W}} \tau_{\eta(k)}^{2}(w) \wedge \bigwedge_{y \in X_{2}} \bigwedge_{e^{*} \in E_{2}}\left(\varphi_{\psi}(f)_{e^{*}}^{\prime}(y) \vee \bigvee_{w \in \mathscr{W}} w_{e^{*}}(y)\right)\right. \\
& \leq \beta \wedge\left(\bigwedge_{w \in \mathscr{W}} \tau_{k}^{1}\left(\varphi_{\psi}^{-1}(w)\right) \wedge \bigwedge_{y \in X_{2}} \bigwedge_{e^{*} \in E_{2}}\left(\varphi_{\psi}(f)_{e^{*}}^{\prime}(y) \vee \bigvee_{w \in \mathscr{W}} w_{e^{*}}(y)\right)\right. \\
& =\left(\beta \wedge \bigwedge_{w \in \mathscr{W}} \tau_{k}^{1}\left(\varphi_{\psi}^{-1}(w)\right) \wedge \bigwedge_{x \in X_{1}} \bigwedge_{e \in E_{1}}\left(f_{e}^{\prime}(x) \vee \bigvee_{w \in \mathscr{W}} \varphi_{\psi}^{-1}(w)_{e}(x)\right)\right. \\
& =\beta \wedge\left(\bigwedge_{g \in \varphi_{\psi}^{-1}(\mathscr{W})} \tau_{k}^{1}(g) \wedge \bigwedge_{x \in X_{1}} \bigwedge_{e \in E_{1}}\left(f_{e}^{\prime}(x) \vee \underset{g \in \varphi_{\psi}^{-1}(\mathscr{W})}{\bigvee} g_{e}(x)\right)\right. \\
& \leq \underset{\mathscr{V} \in 2}{\bigvee} \bigwedge_{\varphi_{\psi}^{-1}(\mathscr{V})} \bigwedge_{x \in X_{1}} \bigwedge_{e \in E_{1}}\left(f_{e}^{\prime}(x) \vee \underset{g \in \mathscr{V}}{\bigvee} g_{e}(x)\right) \\
& =\bigvee_{\mathscr{D} \in 2^{\mathscr{W}}} \bigwedge_{x \in X_{1}} \bigwedge_{e \in E_{1}}\left(f_{e}^{\prime}(x) \vee \bigvee_{w \in \mathscr{D}} \varphi_{\psi}^{-1}(w)_{e}(x)\right) \\
& =\bigvee_{\mathscr{D} \in 2^{\mathscr{W}}} \bigwedge_{y \in X_{2}} \bigwedge_{e^{*} \in E_{2}}\left(\varphi_{\psi}(f)_{e^{*}}^{\prime}(y) \vee \bigvee_{w \in \mathscr{D}} w_{e^{*}}(y)\right) \text {. }
\end{aligned}
$$


From the implication operator properties, we get

$$
\beta \leq\left(\bigwedge_{w \in \mathscr{W}} \tau_{\eta(k)}^{2}(w) \wedge \bigwedge_{y \in X_{2}} \bigwedge_{e^{*} \in E_{2}}\left(\varphi_{\psi}(f)_{e^{*}}(y)^{\prime} \vee \bigvee_{w \in \mathscr{W}} w_{e^{*}}(y)\right)\right) \mapsto \bigvee_{\mathscr{D} \in 2^{(\mathscr{W})}} \bigwedge_{y \in X_{2}} \bigwedge_{e^{*} \in E_{2}}\left(\varphi_{\psi}(f)_{e^{*}}^{\prime}(y) \vee \bigvee_{w \in \mathscr{D}} w_{e^{*}}(y)\right)
$$

Therefore, we obtain the following:

$$
\beta \leq \bigwedge_{\mathscr{W} \subseteq\left(L^{X_{2}}\right)^{E_{2}}}\left\{\left(\bigwedge_{w \in \mathscr{W}} \tau_{\eta(k)}^{2}(w) \wedge \bigwedge_{y \in X_{2}} \bigwedge_{e^{*} \in E_{2}}\left(\varphi_{\psi}(f)_{e^{*}}(y)^{\prime} \vee \bigvee_{w \in \mathscr{W}} w_{e^{*}}(y)\right)\right) \mapsto \bigvee_{\mathscr{D} \in 2^{(\mathscr{W})}} \bigwedge_{y \in X_{2}} \bigwedge_{e^{*} \in E_{2}}\left(\varphi_{\psi}(f)_{e^{*}}^{\prime}(y) \vee \bigvee_{w \in \mathscr{D}} w_{e^{*}}(y)\right)\right\}
$$

This witnesses the desired fact $\beta \leq \operatorname{com}_{\tau^{2}}(\eta(k)$, $\varphi_{\psi}(f)$.

Corollary 3. Let $\left(X_{1}, \tau^{1}\right)$ and $\left(X_{2}, \tau^{2}\right)$ be the L-fuzzy $\left(E_{1}, K_{1}\right)$-soft and L-fuzzy $\left(E_{2}, K_{2}\right)$-soft topological spaces. If the fuzzy soft mapping $\varphi_{\psi, \eta}:\left(X_{1}, \tau^{1}\right) \longrightarrow\left(X_{2}, \tau^{2}\right)$ is surjective, then we have that $\operatorname{com}_{\tau^{1}}\left(k, \widetilde{1}_{X_{1}}\right) \wedge \operatorname{Cont}\left(k, \varphi_{\psi, \eta}\right)$ $\leq \operatorname{com}_{\tau^{2}}\left(\eta(k), \widetilde{1}_{X_{2}}\right)$.
Theorem 11. Let $\left(X_{1}, \tau^{1}\right)$ and $\left(X_{2}, \tau^{2}\right)$ be the L-fuzzy $\left(E_{1}, K_{1}\right)$-soft and L-fuzzy $\left(E_{2}, K_{2}\right)$-soft topological spaces. For the fuzzy soft mapping $\varphi_{\psi, \eta}:\left(X_{1}, \tau^{1}\right) \longrightarrow\left(X_{2}, \tau^{2}\right)$, we have

$$
\operatorname{Con}\left(\eta(k), \varphi_{\psi}(f)\right)^{\prime} \wedge \operatorname{Cont}\left(k, \varphi_{\psi, \eta}\right) \leq \operatorname{Con}(k, f)^{\prime},
$$

for all $k \in K_{1}$ and $f \in\left(L^{X_{1}}\right)^{E_{1}}$.

Proof. Let us choose an arbitrary $\beta \in M(L)$ such that $\beta \triangleleft\left(\operatorname{Con}\left(\eta(k), \varphi_{\psi}(f)\right)^{\prime} \wedge \operatorname{Cont}\left(k, \varphi_{\psi, \eta}\right)\right)$. By Theorems 3 and 4 , the following implications are obtained:

$$
\begin{aligned}
\beta \triangleleft \operatorname{Con}\left(\eta(k), \varphi_{\psi}(f)\right)^{\prime} & =\bigvee\left\{\tau_{\eta(k)}^{2}\left(u^{\prime}\right) \wedge \tau_{\eta(k)}^{2}\left(v^{\prime}\right) \mid \varphi_{\psi}(f) \sqcap u \neq \tilde{0}, \varphi_{\psi}(f) \sqcap v \neq \tilde{0}, \varphi_{\psi}(f) \sqcap u \sqcap v=\tilde{0}, \varphi_{\psi}(f) \nsubseteq u \sqcup v\right\}, \\
\beta \triangleleft \operatorname{Cont}\left(k, \varphi_{\psi, \eta}\right) & =\bigwedge_{h \in\left(L^{X_{2}}\right)^{E_{2}}}\left(\tau_{\eta(k)}^{2}\left(h^{\prime}\right) \mapsto \tau_{k}^{1}\left(\varphi_{\psi}^{-1}(h)^{\prime}\right)\right) .
\end{aligned}
$$

Hence, there exists $u, v \in\left(L^{X_{2}}\right)^{E_{2}}$ which satisfy the conditions $\quad \varphi_{\psi}(f) \sqcap u \neq \widetilde{0}, \varphi_{\psi}(f) \sqcap v \neq \widetilde{0}, \varphi_{\psi}(f) \sqcap u \sqcap v=$ $\widetilde{0}$, and $\varphi_{\psi}(f) \nsubseteq u \sqcup v$ such that $\beta \leq\left(\tau_{\eta(k)}^{2}\left(u^{\prime}\right) \wedge \tau_{\eta(k)}^{2}\left(v^{\prime}\right)\right)$ and also $\beta \leq\left(\tau_{\eta(k)}^{2}\left(h^{\prime}\right) \mapsto \tau_{k}^{1}\left(\varphi_{\psi}^{-1}(h)^{\prime}\right)\right)$, for each $h \in\left(L^{X_{2}}\right)^{E_{2}}$. That is, there exists $u, v \in\left(L^{X_{2}}\right)^{E_{2}}$ satisfying $f \sqcap \varphi_{\psi}^{-1}(u) \neq \widetilde{0}, f \sqcap \quad \varphi_{\psi}^{-1}(v) \neq \widetilde{0}, f \sqcap \varphi_{\psi}^{-1}(u) \sqcap \varphi_{\psi}^{-1}(v)=\widetilde{0}$, and $f \nsubseteq \varphi_{\psi}^{-1}(u) \sqcup \varphi_{\psi}^{-1}(v)$ such that $\beta \leq\left(\tau_{\eta(k)}^{2}\left(u^{\prime}\right) \wedge \tau_{\eta(k)}^{2}\left(v^{\prime}\right)\right)$. And also, $\beta \wedge \tau_{\eta(k)}^{2}\left(h^{\prime}\right) \leq \tau_{k}^{1}\left(\varphi_{\psi}^{-1}(h)^{\prime}\right)$ is satisfied for each $h \in\left(L^{X_{2}}\right)^{E_{2}}$. We conclude that from these observations,

$$
\begin{aligned}
\beta & =\beta \wedge\left(\tau_{\eta(k)}^{2}\left(u^{\prime}\right) \wedge \tau_{\eta(k)}^{2}\left(v^{\prime}\right)\right) \leq \tau_{k}^{1}\left(\varphi_{\psi}^{-1}(u)^{\prime}\right) \wedge \tau_{k}^{1}\left(\varphi_{\psi}^{-1}(v)^{\prime}\right) \\
& \leq \bigvee\left\{\tau_{k}^{1}\left(g^{\prime}\right) \wedge \tau_{k}^{1}\left(h^{\prime}\right) \mid f \sqcap g \neq \widetilde{0}, f \sqcap h \neq \widetilde{0}, f \sqcap g \sqcap h=\widetilde{0}, f \nsubseteq g \sqcup h\right\}=\operatorname{Con}(k, f)^{\prime} .
\end{aligned}
$$

Since the coprime element $\beta$ is arbitrary, we gain the desired inequality for the parameter $k$, $\operatorname{Con}\left(\eta(k), \varphi_{\psi}(f)\right)^{\prime} \wedge$ Cont $\left(k, \varphi_{\psi, \eta}\right) \leq \operatorname{Con}(k, f)^{\prime}$.

\section{Conclusion}

As it is well known, in real life, nothing is described with the help of the 2-valued logic since there is no only black and white in nature. But the idea of fuzzy thinking, which gives some degrees to the phenomena reflect the facts more correctly. In the topological point of view, fuzzy logic, which is also related with quantum mechanics, was applied to the gradation of belongingness and it gave birth to the fuzzy topology. On the other side, the soft set theory which emphasizes the importance/necessity of the parametrization tool for adequate mathematical modeling of the natural facts is one of the preferred tools by the researchers [13, 23-26]. Besides, the combination of these types of sets, named as fuzzy soft set, is one of the adequate tools for modeling. In this respect, we deal with the gradation of the continuity of mappings between fuzzy soft topological spaces (here both of the sets and the axioms of the structure are all fuzzy soft). Since the mappings play the main role to establish the relations between structured sets, the idea proposed here helps 
us to obtain more appropriate and compatible results in such spaces. Despite the theoretical benefits of this method, it is not easy to find numerical examples in application. But this could be overcome by taking a unit interval instead of a lattice.

In relation with the research in this study, notice that soft continuity seems to be the natural tool to prove results more similar to Weierstrass's celebrated theorem. For further research, we hope to investigate this idea and try to find reasonable results. Furthermore, we hope to extend the proposed methods to Pythagorean fuzzy uncertain environments [27] as an additional research.

\section{Data Availability}

The data used to support the findings of this study are cited at relevant places within the text as references and are also available from the corresponding author upon request.

\section{Conflicts of Interest}

The author declares no conflicts of interest.

\section{References}

[1] C. L. Chang, "Fuzzy topological spaces," Journal of Mathematical Analysis and Applications, vol. 24, no. 1, pp. 182-190, 1968.

[2] A. P. Sostak, "Two decades of fuzzy topology: Basic ideas, notions and results," Russian Mathematical Surveys, vol. 44, pp. 1-23, 1989.

[3] C.-Y. Liang and F.-G. Shi, "Degree of continuity for mappings of (L, M)-fuzzy topological spaces," Journal of Intelligent \& Fuzzy Systems, vol. 27, no. 5, pp. 2665-2677, 2014.

[4] B. Pang, "Degrees of continuous mappings, open mappings, and closed mappings in L-fuzzifying topological spaces," Journal of Intelligent \& Fuzzy Systems, vol. 27, no. 2, pp. 805-816, 2014.

[5] Z. Xiu and Q. Li, "Degrees of L-continuity for mappings between L-topological spaces," Mathematics, vol. 7, no. 11, p. 1013, 2019.

[6] D. Molodtsov, "Soft set theory-first results," Computers \& Mathematics with Applications, vol. 37, no. 4/5, pp. 19-31, 1999.

[7] B. Ahmad and A. Kharal, "On fuzzy soft sets," Advances in Fuzzy Systems, vol. 2009, Article ID 586507, 2009.

[8] P. K. Maji, R. Biswas, and A. R. Roy, "Fuzzy soft sets," Journal of Fuzzy Mathematics, vol. 9, no. 3, pp. 589-602, 2001.

[9] P. K. Maji, A. R. Roy, and R. Biswas, "An application of soft sets in a decision making problem," Computers \& Mathematics with Applications, vol. 44, no. 8-9, pp. 1077-1083, 2002.

[10] J. C. R. Alcantud, "An operational characterization of soft topologies by crisp topologies," Mathematics, vol. 9, no. 14, p. 1656, 2021.

[11] T. M. Al-shami, L. D. R. Kočinac, and B. A. Asaad, "Sum of soft topological spaces," Mathematics, vol. 8, no. 6, p. 990, 2020.

[12] T. M. Al-shami and L. D. R. Kocinac, "The equivalence between the enriched and extended soft topologies," Applied and Computational Mathematics, vol. 18, no. 2, pp. 149-162, 2019.

[13] M. K. El-Bably, A. E. F. A. El Atik, and A. El-Atik, "Soft $\beta$ rough sets and their application to determine COVID-19,"
Turkish Journal of Mathematics, vol. 45, no. 3, pp. 1133-1148, 2021.

[14] A. Aygünoğlu, V. Çetkin, and H. Aygün, "An introduction to fuzzy soft topological spaces," Hacettepe Journal of Mathematics and Statistics, vol. 43, no. 2, pp. 197-208, 2014.

[15] V. Çetkin, A. Aygünoğlu, and H. Aygün, "A topological view on application of L-fuzzy soft sets: Compactness," Journal of Intelligent \& Fuzzy Systems, vol. 32, no. 1, pp. 781-790, 2017.

[16] V. Çetkin and H. Aygün, "A topological view on L-fuzzy soft sets: Connectedness degree," Journal of Intelligent \& Fuzzy Systems, vol. 34, no. 3, pp. 1975-1983, 2018.

[17] G. Gierz, A Compendium of Continuous Lattices, SpringerVerlag, Berlin, Germany, 1980.

[18] A. R. Roy and P. K. Maji, "A fuzzy soft set theoretic approach to decision making problems," Journal of Computational and Applied Mathematics, vol. 203, no. 2, pp. 412-418, 2007.

[19] D. Pei and D. Miao, "From soft sets to information systems, granular computing," in Proceedings of the 2005 IEEE International Conference on Robotics and Automation, pp. 617-621, Barcelona, Spain, April 2005.

[20] V. Çetkin and H. Aygün, "On convergence of fuzzy soft filters," in Proceedings of the 3rd International Eurasian Conference on Mathematical Sciences and Applications, pp. 25-28, Vienna-Austria, August 2014.

[21] A. Kharal and B. Ahmad, "Mappings on fuzzy soft classes," Advances in Fuzzy Systems, vol. 2009, Article ID 407890, 2009.

[22] V. Çetkin, "On measures of parameterized fuzzy compactness," Filomat, vol. 34, no. 9, pp. 2927-2938, 2020.

[23] J. C. R. Alcantud, "Softarisons: Theory and practice," Neural Computing and Applications, 2021, In press.

[24] J. Zhan, M. I. Ali, and N. Mehmood, "On a novel uncertain soft set model: Z-soft fuzzy rough set model and corresponding decision making methods," Applied Soft Computing, vol. 56, pp. 446-457, 2017.

[25] J. Zhan and K. Zhu, "A novel soft rough fuzzy set: Z-soft rough fuzzy ideals of hemirings and corresponding decision making," Soft Computing, vol. 21, no. 8, pp. 1923-1936, 2017.

[26] J. Zhan, Q. Liu, and W. Zhu, "Another approach to rough soft hemirings and corresponding decision making," Soft Computing, vol. 21, no. 13, pp. 3769-3780, 2017.

[27] L. Wang, H. Garg, and N. Li, "Pythagorean fuzzy interactive Hamacher power aggregation operators for assessment of express service quality with entropy weight," Soft Computing, vol. 25, no. 2, pp. 973-993, 2021. 